OPEN ACCESS

Edited by: Philipp Albrecht Heinrich Heine University of

Düsseldorf, Germany

Reviewed by:

Michael Dietrich,

Universitätsklinikum Düsseldorf,

Germany

Ermelinda De Meo,

San Raffaele Hospital (IRCCS), Italy

*Correspondence:

Sermin Genc

sermin.genc@deu.edu.tr

${ }^{t}$ These authors have contributed equally to this work

Specialty section: This article was submitted to

Multiple Sclerosis and Neuroimmunology, a section of the journal

Frontiers in Immunology

Received: 06 July 2021 Accepted: 25 October 2021 Published: 10 November 2021

Citation:

Tastan B, Arioz BI, Tufekci KU, Tarakcioglu E, Gonul CP, Genc K and Genc S (2021) Dimethyl Fumarate Alleviates NLRP3 Inflammasome Activation in Microglia and Sickness Behavior in LPS-Challenged Mice.

Front. Immunol. 12:737065. doi: 10.3389/fimmu.2021.737065

\section{Dimethyl Fumarate Alleviates NLRP3 Inflammasome Activation in Microglia and Sickness Behavior in LPS-Challenged Mice}

\author{
Bora Tastan ${ }^{1,2 \dagger}$, Burak I. Arioz ${ }^{1,2 \dagger}$, Kemal Ugur Tufekci ${ }^{1,3}$, Emre Tarakcioglu ${ }^{1,2}$, \\ Ceren Perihan Gonul ${ }^{1,2}$, Kursad Genc ${ }^{4}$ and Sermin Genc ${ }^{1,4^{*}}$
}

${ }^{1}$ Genc Laboratory, Izmir Biomedicine and Genome Center, Izmir, Turkey, ${ }^{2}$ Izmir International Biomedicine and Genome Institute, Dokuz Eylul University, Izmir, Turkey, ${ }^{3}$ Department of Healthcare Services, Vocational School of Health Services, Izmir Democracy University, Izmir, Turkey, ${ }^{4}$ Department of Neuroscience, Health Sciences Institute, Dokuz Eylul University, Izmir, Turkey

NLRP3 inflammasome activation contributes to several pathogenic conditions, including lipopolysaccharide (LPS)-induced sickness behavior characterized by reduced mobility and depressive behaviors. Dimethyl fumarate (DMF) is an immunomodulatory and antioxidative molecule commonly used for the symptomatic treatment of multiple sclerosis and psoriasis. In this study, we investigated the potential use of DMF against microglial NLRP3 inflammasome activation both in vitro and in vivo. For in vitro studies, LPS- and ATP-stimulated N9 microglial cells were used to induce NLRP3 inflammasome activation. DMF's effects on inflammasome markers, pyroptotic cell death, ROS formation, and Nrf2/ NF- $\mathrm{KB}$ pathways were assessed. For in vivo studies, 12-14 weeks-old male BALB/c mice were treated with LPS, DMF + LPS and ML385 + DMF + LPS. Behavioral tests including open field, forced swim test, and tail suspension test were carried out to see changes in lipopolysaccharide-induced sickness behavior. Furthermore, NLRP3 and Caspase-1 expression in isolated microglia were determined by immunostaining. Here we demonstrated that DMF ameliorated LPS and ATP-induced NLRP3 inflammasome activation by reducing $\mathrm{IL}-1 \beta, \mathrm{IL}-18$, caspase-1, and NLRP3 levels, reactive oxygen species formation and damage, and inhibiting pyroptotic cell death in N9 murine microglia via Nrf2/NF- $\mathrm{BB}$ pathways. DMF also improved LPS-induced sickness behavior in male mice and decreased caspase-1/NLRP3 levels via Nrf2 activation. Additionally, we showed that DMF pretreatment decreased miR-146a and miR-155 both in vivo and in vitro. Our results proved the effectiveness of DMF on the amelioration of microglial NLRP3 inflammasome activation. We anticipate that this study will provide the foundation consideration for further studies aiming to suppress NLRP3 inflammasome activation associated with in many diseases and a better understanding of its underlying mechanisms.

Keywords: dimethyl fumarate (DMF), microglia, NLRP3 inflammasome, lipopolysaccharide (LPS), sickness behaviors, pyroptosis 


\section{INTRODUCTION}

Neuroinflammation is a conserved response to all kinds of threats in the central nervous system (CNS) involving certain cell types like neurons, microglia, and astrocytes (1). In acute neuroinflammation, microglia are activated at once, and a rapid response occurs against the dangerous stimuli (2).

Lipopolysaccharide (LPS) -induced sickness behavior is a condition which exhibits depression-like symptoms such as fatigue, cognitive impairment, and limited physical activity (3). A previous study by Aubert et al. showed that LPS is adequate for inducing sickness behavior in mice (4). Numerous proinflammatory cytokines have been proven to be associated with sickness behavior; interleukin-1 (IL-1 $\beta$ ), interleukin-6 (IL-6), and tumor necrosis factor- $\alpha$ (TNF- $\alpha$ ) (5). One important activated pathway is the NLR Family Pyrin Domain Containing Protein 3 (NLRP3) inflammasome during acute neuroinflammation (6).

Inflammasomes, the primary mediators of inflammation, are multiprotein complexes, the activation of which results in the release of inflammatory cytokines and can induce pyroptotic cell death (7). Among the various inflammasomes, NLRP3 inflammasome is the most widely characterized, especially in the CNS $(8,9)$. The NLRP3 inflammasome complex consists of the pattern recognition receptor NLRP3, an adaptor protein called apoptosis-associated speck-like protein (ASC), and the catalytic enzyme caspase-1 in its inactive form. NLRP3 inflammasome complex is activated in two-steps: "priming" and "activation". The priming step involves a danger signal that causes an upregulation in the transcription of NLRP3 and pro-IL- $1 \beta$ and prepares cells to respond to NLRP3 stimulants (10). The most common priming signal is the activation of toll-like receptor 4 (TLR4) by the bacterial product LPS. The presence of LPS causes translocation of the inflammatory transcription factor Nuclear Factor Kappa B (NF-KB) into the nucleus, which upregulates the messenger RNA (mRNA) levels of NLRP3 and pro-IL-1 $\beta(11,12)$. Once there is a sufficient amount of expressed NLRP3 in the cell, multiple stimuli can act as the second "activation" signal. Typical signals include pore-forming crystals, extracellular adenosine 5'-triphosphate (ATP), lysosomal damage, and mitochondrial reactive oxygen species (ROS) (11, 13-15). Upon activation, the NLRP3 inflammasome complex leads to the maturation of the proinflammatory cytokines pro-IL-1 $\beta$ and pro-IL-18. Inflammasome activation may result to further cleavage of Gasdermin D (GSDMD), a pore-forming protein. The formation of pores on the cell membrane results in pyroptotic cell death (16), characterized by cell-swelling, secretion of pro-inflammatory molecules, and the

\footnotetext{
Abbreviations: ATP, Adenosine Triphosphate; ASC, Apoptosis-associated Specklike Protein containing a CARD; CNS, Central Nervous System; DLA, Dimethyl Fumarate and LPS and ATP; DMF, Dimethyl Fumarate; ELISA, Enzyme-Linked Immunosorbent Assay; FBS, Fetal Bovine Serum; GSDMD, Gasdermin D; HMGB-1, High Mobility Group Box 1; IL, Interleukin; Inh, Inhibitor; LA, LPS and ATP; LPS, Lipopolysaccharide; mRNA, Messenger RNA; mtROS, Mitochondrial Reactive Oxygen Species; NF-KB, Nuclear Factor Kappa B; NLRP3, NLR Family Pyrin Domain Containing Protein 3; Nrf2, Nuclear Factor Erythroid 2-related Factor 2; PBS, Phosphate Buffer Saline; PFA, Paraformaldehyde; PI, Propidium Iodide; ROS, Reactive Oxygen Species; RRMS, Relapsing-Remitting Multiple Sclerosis; TNF- $\alpha$, Tumor Necrosis Factor Alfa.
}

propagation of immune response in the CNS (17). Further investigations are required to develop a comprehensive understanding of these dynamic processes and better therapeutic strategies for disorders associated with NLRP3 inflammasome.

Dimethyl Fumarate (DMF) is a drug approved by the Food and Drug Administration (FDA) for Relapsing-Remitting Multiple Sclerosis (RRMS) since the early 2010s (18). Two different clinical trials (DEFINE and CONFIRM) demonstrated that DMF intake could lead to a decline in brain lesions and decreased relapse rates in RRMS patients $(19,20)$. In vitro and in vivo studies have proven that DMF possesses immunomodulatory (21), antiinflammatory (22), anti-oxidative, and neuroprotective properties (23). The protective nature of DMF has been attributed to the activation of the Nuclear Factor Erythroid 2-related Factor 2 (Nrf2), as DMF is considered to be the most clinically developed Nrf2 activator (24). Once activated, Nrf2 upregulates anti-oxidative and cytoprotective Antioxidant Responsive Element (ARE)containing genes (25). DMF also inhibits the pro-inflammatory pathway NF- $\mathrm{KB}$, thus downregulating the expression of NLRP3 and pro-IL-1 $\beta$ and decreases inflammatory response (26). It has previously been shown that DMF suppresses NLRP3 inflammasome in THP-1 cells and the dextran sulfate sodiuminduced experimental colitis model $(27,28)$. However, DMF's protective effects and DMF's mechanism of action on microglial NLRP3 inflammasome activation remain largely unexplored, since there are currently no relevant experimental study available.

In the present study, we aimed to investigate DMF's effects on microglial NLRP3 inflammasome activation using both in vitro and in vivo models. We hypothesized that DMF would exert protective effects against NLRP3 inflammasome activation and inflammasome-induced pyroptosis in microglia. Herein, we demonstrated that DMF alleviated microglial NLRP3 inflammasome activation via modulation of NF- $\mathrm{\kappa B}$ and Nrf2, and ameliorated pyroptotic cell death. Furthermore, our in vivo study on mice revealed that DMF intake restored LPS-induced sickness behavior. Taken together, these results indicate that DMF treatment prevented NLRP3 inflammasome activation in both in vitro and in vivo models.

\section{MATERIALS AND METHODS}

\subsection{Chemicals and Reagents}

DMF, adenosine 5'-triphosphate disodium hydrate, LPS (LPS 055: B5), ML385, Fetal bovine serum (FBS), RPMI 1640 cell culture media, L-Glutamine, penicillin/streptomycin, phosphate-buffered saline (PBS), and trypsin/EDTA were purchased from SigmaAldrich (St. Louis, USA). Ultra-Pure LPS (LPS 0111: B4) were purchased from In vivoGen (San Diego, USA). Supplementary Table 1 contains all antibodies used in this research.

\subsection{Cell Culture}

Murine N9 microglia were provided by Dr. Paola RicciardiCastagnoli (Cellular Pharmacology Center, Milan, Italy) (29). Cells were sustained in RPMI 1640 containing 10\% FBS and 2 $\mathrm{mM} \mathrm{L-Glutamine,} 100 \mathrm{U} / \mathrm{ml}$ penicillin, and $100 \mu \mathrm{g} / \mathrm{ml}$ streptomycin at $37^{\circ} \mathrm{C}$ with $5 \% \mathrm{CO}_{2}$. Treatments were 
introduced as follows unless a different experimental setup is implemented; DMF $(10 \mu \mathrm{M})$ for 1 hour, LPS $(1 \mu \mathrm{g} / \mathrm{ml})$ for 4 hours, and ATP (5 mM) for 1 hour.

\subsection{LDH Assay}

The cytotoxicity of N9 microglia was determined by checking $\mathrm{LDH}$ release with Cytotoxicity Detection $\mathrm{Kit}^{\text {PLUS }}$ (Roche, Germany) by following the manufacturer's protocol. Microplate reader Varioskan (Thermo, USA) was used to obtain the colorimetric change of cells at $492 \mathrm{~nm}$ with a reference wavelength of $630 \mathrm{~nm}$. Cytotoxicity percentages were calculated as follows: Cytotoxicity $=(\mathrm{OD}$ Sample - OD Low Control) (OD Maximal Release -OD Low Control) x 100

\subsection{Cell Viability Assay}

According to the manufacturer's protocol, N9 microglia viability was investigated with Cell Counting Kit-8 (Sigma Aldrich, St. Louis, USA). Microplate reader Varioskan (Thermo, USA) was used to determine the colorimetric change of cells at $450 \mathrm{~nm}$ with a reference wavelength of $630 \mathrm{~nm}$. Cell viability percentages was calculated as the percentage of untreated cells.

\subsection{ELISA}

Secreted IL-1 $\beta$ and IL-18 levels were determined by IL-1 $\beta$, and IL18 sandwich enzyme-linked immunosorbent assay (ELISA) kits (Invitrogen, USA) according to the manufacturer's instructions.

\subsection{Real-Time qRT-PCR}

Total RNA was extracted by using the Nucleospin RNA II Kit (Macherey-Nagel, Germany) according to the manufacturer's protocol. Total RNA concentration was measured by the Nanodrop spectrophotometer (Thermo Fischer, USA), and 1 $\mu \mathrm{g}$ of RNA was used for reverse transcription with cDNA RevertAid First Strand cDNA Synthesis Kit (Thermo Scientific, USA) by following the manufacturer's protocol. GoTaq ${ }^{\circledR}$ qPCR Master Mix (Promega, USA) was used for performing quantitative realtime PCR with LightCycler ${ }^{\circledR} 480$ Instrument II (Roche Life Science, USA) according to the manufacturer's protocol.

For miRNA studies, RNA was isolated by the miRNeasy mini kit (Qiagen, Germany). miScript II RT Kit and QuantiTect SYBR Green PCR Kits (Qiagen, Germany) were used for cDNA synthesis and qPCR, respectively. All the primers utilized in experiments are listed in Supplementary Table 2. Relative expression levels were calculated with the $2^{-\Delta \Delta \mathrm{Ct}}$ method.

\subsection{Protein Extraction and Western Blot Analysis}

Protein extraction and western blot analysis were carried out as described before (30). Briefly, RIPA lysis buffer (50mM TrisHCL, pH 7.4, 150mM NaCl, 0.25\% deoxycholic acid, $1 \%$ Nonidet P-40, $1 \mathrm{mM}$ EDTA) supplemented with protease and phosphatase inhibitor (Thermo Scientific, Massachusetts, USA) was used for total protein isolation from both cell lysate and supernatant. NE-PER, Nuclear and Cytoplasmic Extraction Reagents (Thermo Scientific, USA) were used to isolate nuclear and cytosolic fractions. For Western Blot, equal amounts of protein samples were run on a $8-15 \%$ SDS-PAGE gel depending on the protein of interest and then was transferred to the PVDF membrane. Depending on the antibodies used, the PVDF membrane was blocked with $5 \%$ BSA or $5 \%$ dry milk in TBST. The membrane was incubated with a primary antibody overnight at $4^{\circ} \mathrm{C}$ and a secondary antibody for 1 hour at room temperature. At the end of incubations, antigen-antibody complexes were screened with Luminata Forte Western HRP substrate (Merck Millipore, USA) using a densitometer (Vilber Lourmat Gel Imager System, CA). ImageJ software (National Institutes of Health, USA) was used for band density analysis (27). Results were normalized to $\beta$-actin and Lamin $A / C$ for quantification or given as arbitrary unit (a.u.) for secreted proteins present in the supernatant.

\subsection{Caspase-1 Activity Assay}

Luminometric Caspase-Glo ${ }^{\circledR} 1$ Inflammasome Assay (Promega, USA) was used for measuring caspase- 1 activity by following the manufacturer's protocol. Centro XS3 lb 960 microplate luminometer (Berthold Technologies, Germany) was used to measure the luminescence of cells. Caspase-1 percentages were calculated as the percentage of untreated cells.

\subsection{Propidium lodide Staining for Pyroptosis Detection}

Cells were treated with $50 \mu \mathrm{g} / \mathrm{ml}$ propidium iodide (PI) stain (Thermo Scientific, USA) in the last 15 minutes of ATP treatment. Fluorescent images were captured using the fluorescent microscopy system (Olympus IX-71, Japan). PI-positive and negative cells were counted by ImageJ software (National Institutes of Health, USA) (31). PI-positive cell percentages were calculated by comparing them to untreated cells.

\subsection{Mitochondrial ROS Measurement With MitosoX}

Mitochondrial ROS (mtROS) was determined with the MitoSOX reagent. After treatment, microglia were treated with $5 \mu \mathrm{M}$ MitoSOX (Invitrogen, USA) for 15 minutes at $37^{\circ} \mathrm{C}$, and fluorescence absorbance values were measured at $530 \mathrm{~nm}$ with a reference wavelength of $590 \mathrm{~nm}$ with the microplate reader Varioskan (Thermo scientific, Massachusetts, USA). For immunofluorescence assay, cells were treated with $5 \mu \mathrm{M}$ MitoSOX for 15 minutes at $37^{\circ} \mathrm{C}$ and $0.1 \mu \mathrm{M}$ DAPI for 2 minutes at $37^{\circ} \mathrm{C}$. Images were obtained under an inverted fluorescent microscope, Olympus IX-71 (Olympus, Japan).

\subsection{Intracellular ROS Measurement With DCFDA}

Total intracellular ROS was investigated with CM-H2DCFDA (Invitrogen, USA) according to the manufacturer's instructions. The fluorometric measurements were done at $495 \mathrm{~nm}$ with a reference wavelength of $527 \mathrm{~nm}$ with a Varioskan Flash (Thermo Scientific, USA) microplate reader.

\subsection{Mitochondria Membrane Potential Measurement}

JC-1 (Thermo, USA) stain was used for investigating mitochondria membrane potential measurement of microglia 
according to the manufacturer's instructions. FACS Canto II analyzer (Becton Dickinson, USA) using a $488 \mathrm{~nm}$ laser (Becton Dickinson, USA) was used for assessing the mitochondria membrane potential.

\subsection{Animals and Experimental Design}

All the animal experiments and animal care were strictly performed according to the Izmir International Biomedicine and Genome Institute Local Ethic Committee for Animal Experiments (IBG-AELEC), protocol number: 03/2017. For in vivo study, male $\mathrm{BALB} / \mathrm{c}$, aged 12-14 weeks, were used. All the animals were housed and maintained in IBG-Vivarium under controlled conditions $\left(22 \pm 2^{\circ} \mathrm{C} ; 12\right.$ hours light/dark periods) with access to food and water ad libitum. In the experimental setup, mice were grouped randomly into four different groups: Control, LPS, DMF + LPS, and ML385 + DMF + LPS ( $\mathrm{n}=7$ mice/group). Before the experimental procedures, all mice were weighed and controlled. Mice were intraperitoneally injected with ML385 (30 mg/kg) 24 hours before LPS injection, DMF $(30 \mathrm{mg} / \mathrm{kg}) 1$ hour before LPS injection, and LPS (5 mg/kg). After 24 hours from LPS injection, all animals proceeded with the clinical scoring, weighing, and behavioral tests. Animals were sacrificed by decapitation, and their brains were collected.

\subsection{Clinical Evaluation and Behavioral Tests}

To assess DMF's effects on sickness behaviors, the following evaluations and behavioral tests were carried out.

\subsubsection{Clinical Scoring and Weighing}

Sickness behaviors and bodyweight were scored and weighed by a blinded observer. To detect the change in body weight, animals were weighed before and after injections. Clinical scoring was carried out based on a 4-point scale, as described before (32). Accordingly, each symptom (lethargy, ptosis, and huddling) adds 1 point to the overall score. The absence of symptoms was accepted as 0 .

\subsubsection{Open Field Test}

OFT was carried out to assess sickness behavior. At the end of treatments, animals were gently put in the corner of plastic boxes $(40 \mathrm{~cm} \times 40 \mathrm{~cm} \times 40 \mathrm{~cm}$ ) whose floors were divided into equal squares. Their free movement was observed for 6 minutes. The number of squares crossed by animals was recorded and counted. The boxes were cleaned after each test with $70 \%$ ethanol to eliminate any sign of odor.

\subsubsection{Tail Suspension Test}

After OFT, TST was performed. After treatments, each animal was hanged individually by adhesive tape separated by opaque fences. The tape was placed $1 \mathrm{~cm}$ from the tip of their tail. The behaviors of animals were monitored for a period of 6 minutes. Animals are considered immobile when they stay motionless for 5 seconds, and their immobility time was recorded.

\subsubsection{Forced Swim Test}

Lastly, FST, a commonly used behavioral test for sickness behavior, was carried out as described previously (30). Briefly, an empty glass cylinder ( $30 \mathrm{~cm}$ height, $10 \mathrm{~cm}$ diameter) filled up to $25 \mathrm{~cm}$ was used for the tests, and the temperature of the water used was at $25 \pm 1^{\circ} \mathrm{C}$. The animals were placed into this cylinder and monitored for 6 minutes. The latency to the immobility in which animals float in the water without moving for at least 5 seconds was recorded for each animal. Water was changed after each FST test.

\subsection{Microglia Isolation}

Microglia isolation was performed as previously described (33). Briefly, animals were anesthetized and transcardially perfused with ice-cold PBS using a peristaltic pump. After removing meninges, brains were dissected and collected in cold buffer HBSS (Hanks' balanced salt solution, Sigma, USA) containing 1\% BSA and 1mM EDTA. Brains were chopped in a petri dish containing HBSS with the help of a razor blade and then filtered through a $70 \mu \mathrm{m}$ cell strainer into a $50 \mathrm{ml}$ falcon tube. Collected samples were centrifuged for 12 minutes at $300 \mathrm{xg}$ and $10^{\circ} \mathrm{C}$. The pellets were resuspended in $5 \mathrm{ml}$ cold $37 \%$ Percoll in PBS, underlaid with $4 \mathrm{ml} \mathrm{70 \%} \mathrm{Percoll} \mathrm{and}$ overlayed with $4 \mathrm{ml} \mathrm{30 \%} \mathrm{Percoll} \mathrm{in} \mathrm{a} 15 \mathrm{ml}$ Falcon Tube. These falcons were centrifuged for 40 minutes at $600 \mathrm{x}$ g and $10^{\circ} \mathrm{C}$ with 0 acceleration and 0 brakes. Finally, the cell layer was collected from the $70 \%$ and $37 \%$ Percoll interface, transferred into an equal volume of PBS containing 1\% FBS, and centrifuged for 5 minutes, $400 \mathrm{x} \mathrm{g}$, $4^{\circ} \mathrm{C}$. The supernatant was discarded carefully, and $400 \mu \mathrm{l}$ PBS was added to the tube, and cells were used in further experiments.

\subsection{Immunofluorescence Staining}

For immunostaining (ASC speck, NF- $\kappa B$, and Nrf2 staining), cells were fixed with $4 \%$ paraformaldehyde (PFA) at $37^{\circ} \mathrm{C}$ for 15 minutes, then washed with PBS. Permeabilization and blocking were done using PBS containing 10\% Goat Serum and 0.5\% Triton-X-100 at $37^{\circ} \mathrm{C}$ for 30 minutes. Cells were incubated with primary antibody overnight, and the following day, the secondary antibody was incubated for 1 hour. Images were acquired with LSM 880 Confocal microscopy (Zeiss, Germany) or fluorescent microscope Olympus BX-61 (Olympus, Japan).

For primary microglia staining, isolated cells were centrifuged for 4 minutes, 1600 RPM at RT, fixed with $4 \%$ PFA for 15 minutes at $37^{\circ} \mathrm{C}$, then washed 1-2 times with PBS, and permeabilized with PBS containing \%0,1 Triton-x100 for 10 minutes at RT. After washing twice, cells were blocked with PBS containing 5\% donkey serum for 30 minutes at RT and incubated for an overnight hour at $4^{\circ} \mathrm{C}$ with primary antibody IBA1 and NLRP3/Caspase-1 diluted in PBS. On the next day, the cells were rewashed with PBS twice and incubated with secondary antibody diluted in PBS for 1 hour at RT. In the last 20 minutes of incubation, Hoechst was added for counterstaining. In the end, the cells were washed PBS twice, and slides were mounted. Visualization of the slides was done using a fluorescent microscope Olympus BX-61 (Olympus, Japan).

All images were analyzed with ImageJ software (National Institutes of Health, USA). In the analysis of ASC speck images, ASC speck positive cells were counted, and data were given as the percentage of total cells. Image analysis was conducted bling to avoid experimental bias. 


\subsection{Statistical Analysis}

Graphpad Prism 8.0 (Graphpad Software Inc., CA, USA) was used for all Statistical analyses. Shapiro-Wilk test was followed for the normality test. Accordingly, Mann-Whitney U-test and Student's t-test were utilized for non-parametric and parametric data, respectively. For comparison of multiple groups with normal distribution, one-way ANOVA with Bonferroni multiple comparison corrections was used. On the other hand, for the comparisons with three or more group without normal distribution, Kruskal-Wallis test with Dunn's multiple comparison was utilized. All data were provided as mean \pm standard error of the mean. A p-value less than $0.05(\mathrm{p}<0.05)$ was considered as statistically significant in all experiments. All in vitro experiments were repeated three times, unless otherwise indicated.

\section{RESULTS}

\subsection{DMF Reduced NLRP3 Inflammasome Related Cytokines IL-1 $\beta$ and IL-18 at Both mRNA and Protein Levels in N9 Cells}

Ameliorative effects of DMF on secreted NLRP3 inflammasomerelated cytokines IL-1 $\beta$ and IL-18 were tested with ELISA. We found that DMF significantly decreased the amounts of IL-1 $\beta$ (Figure 1A) and IL-18 (Figure 1B), which were induced by LPS and ATP treatment. Next, expression levels of IL-1 $\beta$ and IL-18 were evaluated with the RT-qPCR method. More than a 3 -fold significant decline in IL-1 $\beta$ mRNA level (Figure 1C) and approximately 2 -fold significant decline in IL-18 mRNA level (Figure 1D) were observed with DMF pretreatment. Furthermore, we observed that DMF significantly reduced both pro-IL-1 $\beta$ (Figures 1E, F) and secreted mature IL-1 $\beta$ protein levels (Figures 1E, G) against NLRP3 inflammasome activation. Additionally, we found that DMF significantly decreased High Mobility Group Box 1 (HMGB1) expression in LPS and ATPinduced microglia (Figures 1E, H).

\subsection{DMF Decreased NLRP3, Caspase-1, and ASC, Which Forms the NLRP3 Protein Complex in N9 Cells}

Next, we investigated DMF's effect on the activated NLRP3 protein complex consisting of NLRP3, ASC adaptor protein, and Caspase-1. The intracellular level of p45 caspase-1 protein showed no significant difference in all three groups (Figures 2A, B). Active caspase-1 (p20) protein level significantly decreased in DMF pretreated cells compared to LPS and ATP-induced cells (Figures 2A, C). Additionally, Caspase-1 activity was evaluated and, there was a 2 -fold increase with LPS and ATP treatment as it decreased 25\% with DMF pretreatment (Figure 2D). Next, our investigations demonstrated that the DMF pretreated group showed an approximately 2 -fold significant reduction in protein (Figures 2E, F) level and more than 2.5-fold significant reduction of the NLRP3 mRNA level (Figure 2G) as compared to the LPS and ATP treated group. Adaptor protein ASC is critically essential for the assembly of the NLRP3 inflammasome complex.
Immunofluorescence staining results indicated that DMF diminished ASC speck formation induced by LPS and ATP administration (Figures 2H, I).

\subsection{DMF Protected Against Pyroptotic Cell Death and Prevented Cleavage of GSDMD in N9 Cells}

We further checked the effects of DMF on pyroptotic cell death stimulated by LPS and ATP administration. In the LDH assay (Figure 3A), we observed a significant decrease in cell death with DMF pretreatment compared to microglia with LPS and ATP treatment. Additionally, we revealed significant augmentation in microglia viability with DMF pretreatment (Figure 3B) We further carried out PI staining, a DNA intercalating dye that stains pyroptotic cells. We found a significant decrease in the number of pyroptotic cells with DMF pretreatment in PI staining (Figures 3C, D). Lastly, we evaluated the effect of DMF on GSDMD cleavage with western blotting and found a significant decline in cleaved GSDMD levels in DMF pretreated group against only the LPS and ATP treated group (Figures 3E, F).

\subsection{DMF Prevented Mitochondrial and Total ROS Production and Enhanced Mitochondrial Membrane Potential In Vitro}

We studied mitochondrial ROS production with MitoSOX. Significantly elevated mtROS levels induced by LPS and ATP treatment were significantly reduced with DMF pretreatment (Figures 4A, B). After that, we investigated intracellular total ROS production of microglia with DCFDA. DMF pretreatment significantly alleviated, nearly 2.5 -fold, total ROS production compared to the LPS, and ATP treated group (Figure 4C). Lastly, we checked the mitochondrial membrane potential with JC-1 staining. There was a significant decrease in the green ratio in DMF pretreated group compared only to the LPS, and ATP treated group via flow cytometry (Figures 4D, F); green signal indicates deteriorated mitochondrial membrane potential, whereas the red signal denotes healthy mitochondrial membrane potential. Furthermore, we confirmed the improvement of mitochondrial membrane potential with DMF pretreatment under a fluorescent microscope with JC-1 staining. We observed an increased green signal with LPS and ATP introduction and, conversely, an increased red signal and reduced green signal with DMF pretreatment (Figure 4E).

\subsection{DMF Ameliorated NF- $\kappa B$ Activation and Reduced miR-155/146a Expression}

As the NF- $\kappa B$ pathway is the upstream pathway of the NLRP3 inflammasome pathway, we evaluated DMF's effect on the NF$\kappa \mathrm{B}$ pathway with western blotting and immunostaining. Firstly, we found that DMF significantly increased the I $\kappa \mathrm{B}-\alpha$ protein level, the expression of which decreased by LPS treatment (Figures 5A, B). Next, we checked DMF's effects on NF- $\mathrm{BB}$ subunits p65 and p50. We demonstrated that DMF significantly reduced nuclear p-p65 compared to the LPS treated group 
A

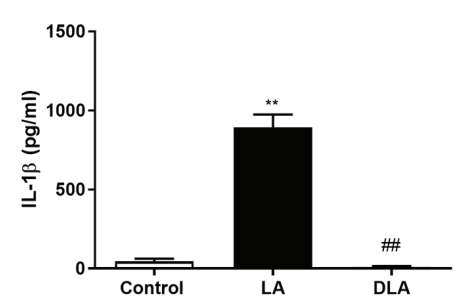

D

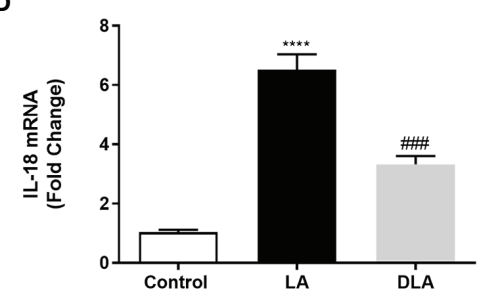

G

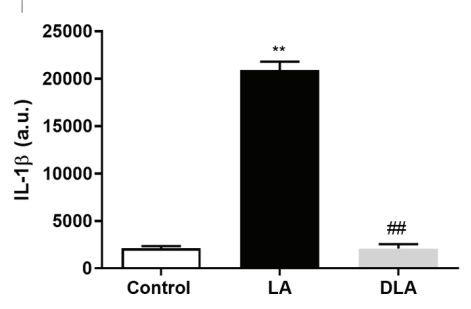

B

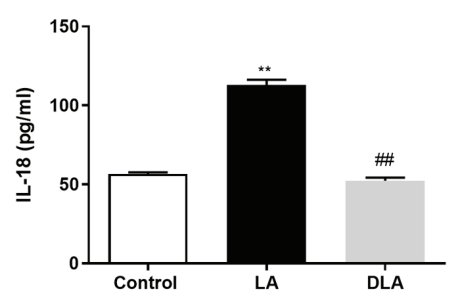

E

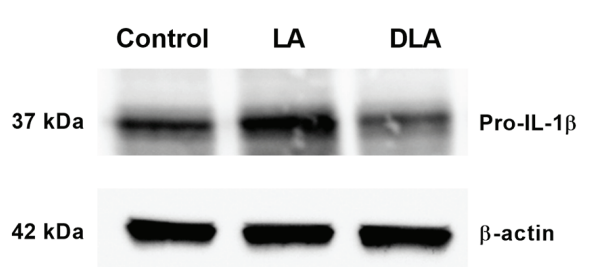

H

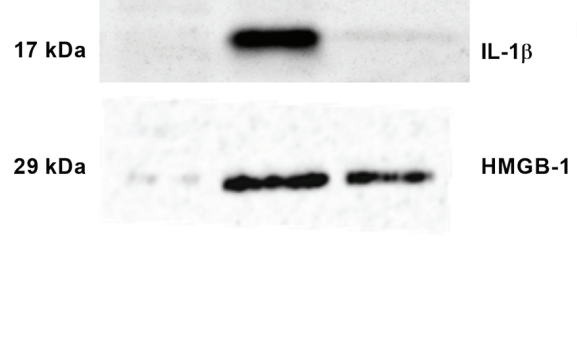

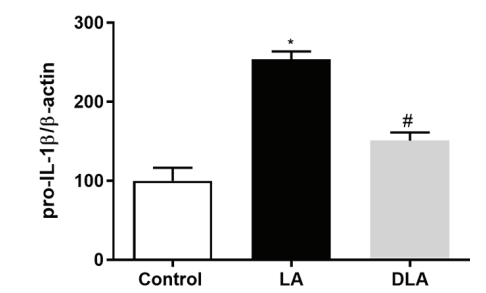
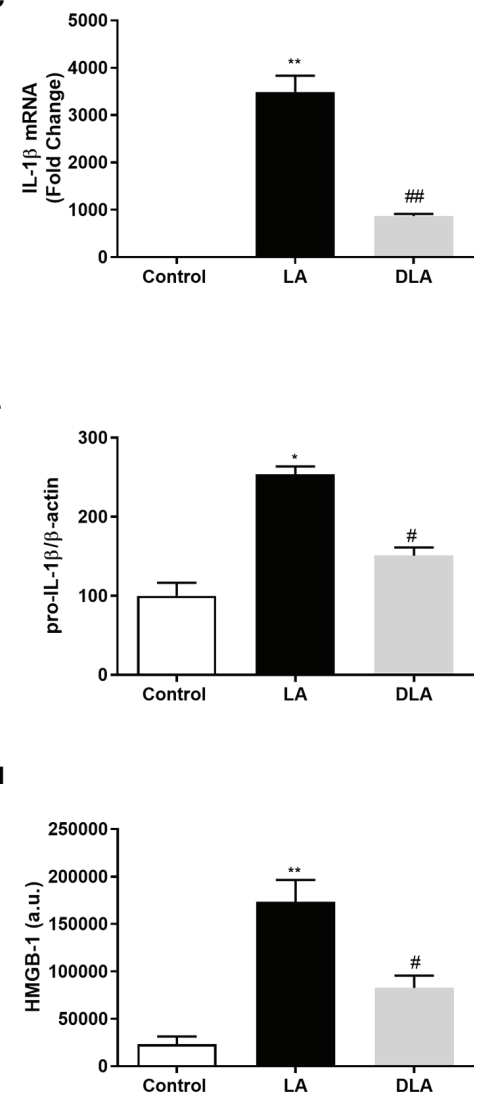

FIGURE 1 | DMF suppressed inflammasome-induced secretion of pro-inflammatory cytokines and HMGB-1. N9 microglia were pretreated with DMF (10 $\mu M$ ) for 1hour, primed with UP-LPS (1000 ng/ml) for 4 hours, and followed by ATP $(5 \mathrm{mM})$ for 1 hour. (A, B) Pretreatment with DMF decreased the protein and mRNA

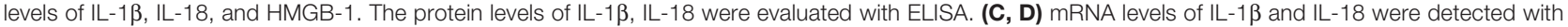
qRT-PCR. (E-H) The expression of pro-IL-1 $\beta$, IL-18, and their secretion along with HMGB-1 in supernatants were assessed by Western blot. Data are presented as mean \pm S.E.M, $n=5 .{ }^{*} p<0.05,{ }^{* *} p<0.01,{ }^{* \star * *} p<0,0001$ compared to control and ${ }^{\#} p<0.05,{ }^{\# \#} p<0.01,{ }^{\# \# \#} p<0,001$ compared to LPS and ATP induced cells.

(Figures 5A, C). Furthermore, DMF pretreatment significantly reduced increased protein levels of nuclear p65 and p50 reduced with LPS treatment (Figures 5A, D, E). We also confirmed our result with immunostaining against the p65 and the p50 subunit of NF- $\kappa B$ as DMF decreased $\mathrm{p} 50$ and p 65 translocation into the nucleus (Figures 5F, G). Lastly, we further checked the expression levels of miR-155 and miR-146a with the RT-qPCR method, as these miRNAs are known to act upstream of NF- $\mathrm{KB}$ and be related to inflammasome activation. We showed that DMF significantly decreases the expression levels of miR-155 (Figure 5H) and miR-146a (Figure 5I) in both in vitro and in vivo models of NLRP3 inflammasome activation.

\subsection{DMF Induced Nrf2 Translocation and Modulated NLRP3 Inflammasome Activation via Nrf2 in N9 Cells and LPS-Challenged Mice}

Given that DMF is a well-known activator of the Nrf2 signaling pathway, we further checked DMF's effect on Nrf2 on microglia.
First, we showed that DMF induced Nrf2 activation and led to the translocation of Nrf2 to the nucleus (Figures 6A-C). Next, we determined the expression levels of Nrf2 target genes, including Ho-1, Nqo1, Gclc, Gclm, Srxn1, and its inactivator Keap-1 by RT-qPCR. The results demonstrated that treatment with DMF increased the expression levels of the target genes and decreased Keap-1 expression (Figure 6D). We further confirmed our results with an in vivo study. In particular, microglia isolated from mice were stained against NLRP3 and Caspase-1. The results demonstrated that DMF decreased NLRP3 and Caspase- 1 protein expression in those mice injected with DMF. However, ML385, a Nrf2 inhibitor, reversed this effect and increased the expression levels showing that Nrf2 mediates the protective effects of DMF (Figures 6E-H).

\subsection{DMF Restored Sickness Behaviors in LPS-Challenged Mice}

In order to assess the effects of DMF on LPS-induced sickness behaviors, behavioral tests and clinical evaluation on mice were 
A

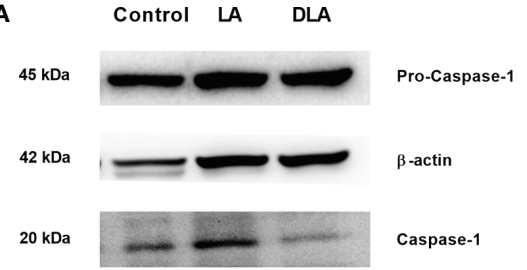

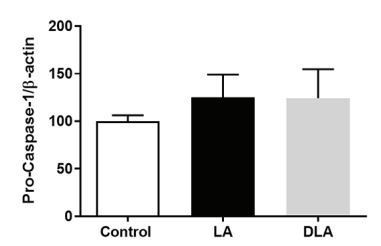

C

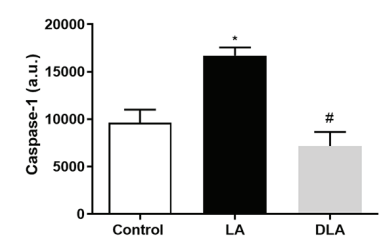

D

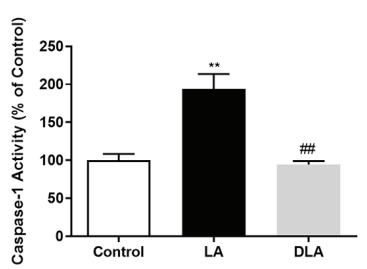

H

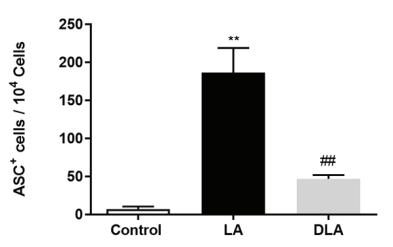

E

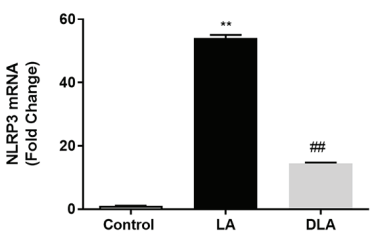

F

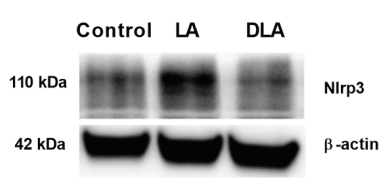

G

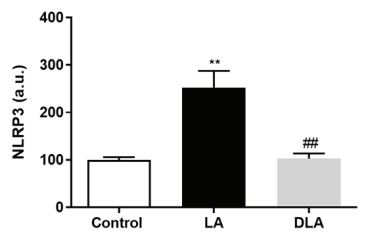

I

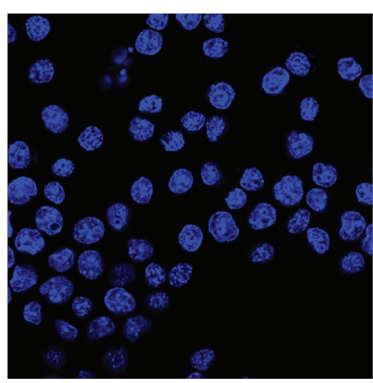

LA

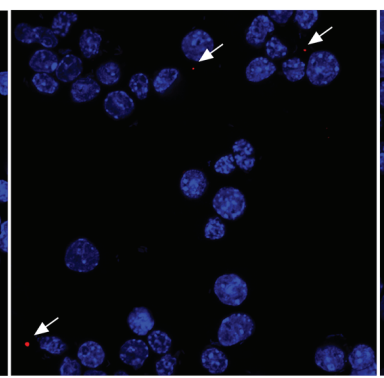

DLA

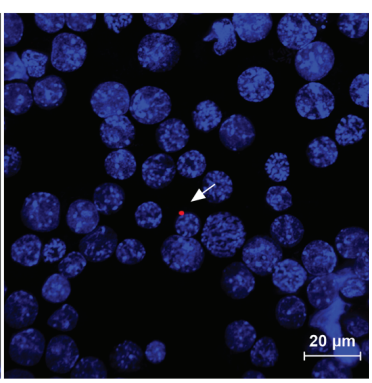

FIGURE 2 | DMF decreased the expression of inflammasome complex proteins, prevented caspase-1 activity and ASC speck formation. N9 microglia were pretreated with DMF $(10 \mu \mathrm{M})$ for $1 \mathrm{~h}$, primed with UP-LPS $(1000 \mathrm{ng} / \mathrm{ml})$ for 4 hours, and followed by ATP $(5 \mathrm{mM})$ for 1 hour. Pretreatment with DMF led to decrease expression of NLRP3, inhibited cleavage of pro-caspase 1(p45) into caspase-1(p20), and prevented caspase-1 activity and ASC speck formation in microglia. (A-C) The expression of pro-caspase-1 and its cleavage were evaluated with Western blot. (D) Caspase-1 activity was measured with a luminometric assay. (E) mRNA expression of NLRP3 was measured with qRT-PCR. (F, G). Protein expression of NLRP3 was detected by Western blot. (H, I) ASC speck formation was demonstrated by the IF method (Blue staining - Hoechst, Red staining - ASC). Data are presented as mean \pm S.E.M, $n=5 .{ }^{*} p<0.05,{ }^{* \star} p<0.01$ compared to control and $\# p<0.05, \# p<0.01$ compared to LPS and ATP induced cells.

carried out (Figure 7A). In this regard, we conducted clinical scoring, weighed the mice, and performed OFT, FST, and TST. Results demonstrated that injection of LPS resulted in sickness behaviors in $\mathrm{BALB} / \mathrm{c}$ mice while treatment with $\mathrm{DMF}$ reversed this situation (Figure 7B). On the other hand, pretreatment with Nrf2 inhibitor, ML385, reversed DMF's protective effects on the LPS-induced sickness phenotype. We also weighed the animals before and after treatment. Results indicated that treatment with DMF decreased weight loss and inhibition of Nrf2 activation worsened this situation (Figure 7C). Next, we performed behavioral tests. In OFT, the animals treated with DMF crossed a significantly higher number of squares compared to LPS. However, the ones injected with ML385 traveled less in the open field (Figure 7D). Again, in TST and FST, DMF increased the latency to immobility. These effects also seem dependent on Nrf2 activation as the use of ML385 decreased the latency to immobility (Figures 7E, F). As a result, DMF significantly restored sickness behaviors, reversed clinical course, and prevented weight loss induced by LPS.

\section{DISCUSSION}

In the present study, we proved that DMF ameliorated microglial NLRP3 inflammasome activation via the NF- $\kappa \mathrm{B} / \mathrm{Nrf} 2$ axis. The NLRP3 inflammasome activation is a fundamental part of the innate immune system, and its excessive activation leads to sterile inflammation (34). Mounting evidence suggests the involvement of inflammasome during LPS-induced sickness behavior. LPS reduces mobility and locomotion and increases pro-inflammatory cytokines like IL- $1 \beta$ and IFN-gamma in rats; these are characteristics of sickness behavior (35). Other cytokines like TNF- $\alpha$ and IL- 6 were also increased by LPS administration in rodents (36). Our immunohistochemical 
A

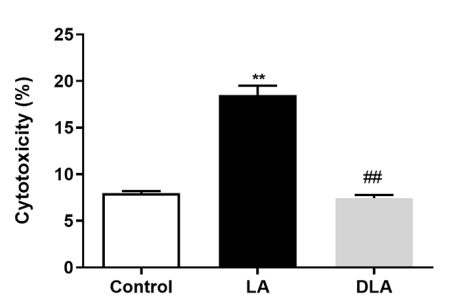

D

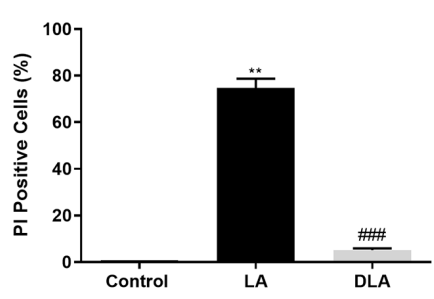

B

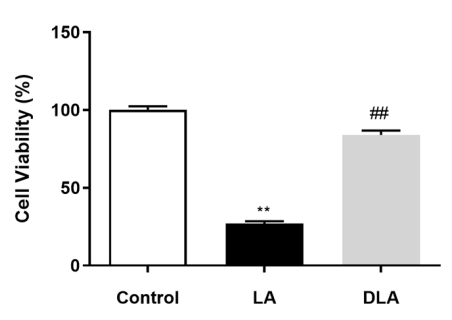

E

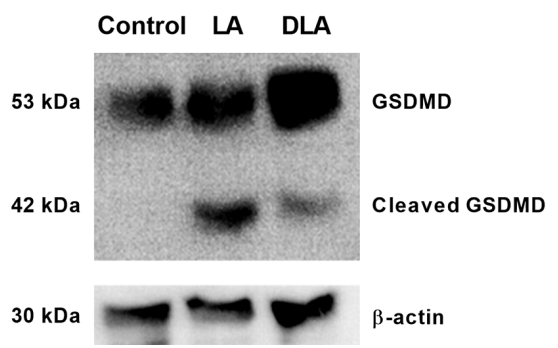

BF

\section{C}

일
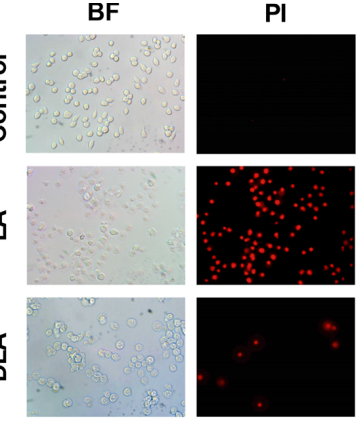

MERGED

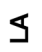
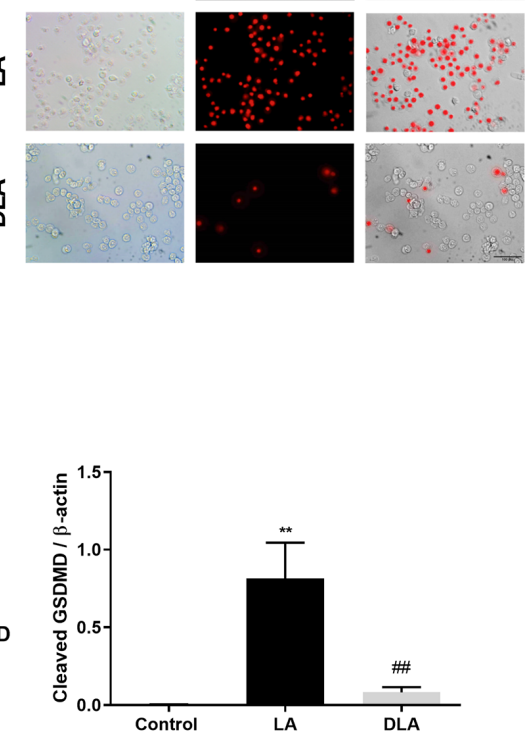

FIGURE 3 | DMF attenuated GSDMD cleavage and subsequent pyroptotic cell death. N9 microglia were pretreated with DMF (10 $\mu$ M) for 1h, primed with UP-LPS $(1000 \mathrm{ng} / \mathrm{ml})$ for 4 hours, and followed by ATP $(5 \mathrm{mM})$ for 1 hour. DMF pretreatment inhibited NLRP3 inflammasome-induced GSDMD cleavage and pyroptotic cell death in N9 microglia. (A, C, D) Changes in pyroptotic cell death were evaluated with the LDH assay and PI staining (Red staining - PI). (B) The effect of DMF on cell viability was measured with the CCK-8 assay. (E, F) GSDMD expression and its cleavage were detected with Western blot. Data are presented as mean \pm S.E.M, $\mathrm{n}=5 .{ }^{\star \star} \mathrm{p}<0.01$ compared to control and ${ }^{\# \#} \mathrm{p}<0.01,{ }^{\# \# \#} \mathrm{p}<0.01$ compared to LPS and ATP induced cells.

analysis of microglia cells obtained from LPS-treated animals confirmed the presence of NLRP3 inflammasome activation. Our results (Supplementary Table 3) support that the activation of the NLRP3 inflammasome is involved in the pathogenesis of LPS-induced sickness behavior, and inhibition of NLRP3 inflammasome might represent a potential therapeutic strategy against inflammatory diseases.

In our study, we utilized the N9 microglial cell line in the in vitro assays. The N9 microglia were originally derived from the mouse brain, since previous studies confirmed that N9 shares standard features with primary microglia in morphology, immune response, and phagocytosis ability (29). Further studies also revealed that N9 cells can elicit the required response against inflammatory stimuli such as Amyloid- $\beta$, TNF- $\alpha$, and IL-1 $\beta$ (37). Thus, N9 cell line has been extensively used in microglia research.

DMF is an FDA-approved Nrf2 activator and has been used safely in the treatment of RRMS. The neuroprotective (38), antioxidative (39), and anti-inflammatory (40) effects of DMF in the brain have been well documented in previous studies. In a recent study, where a rat model of chronic unpredictable mild stress was used, DMF restored sickness behaviors via the axis of MAPK/ ERK1/2 and JNK pathways (41). However, to the best of our knowledge, there has been no study on the role of DMF in the regulation of NLRP3 inflammasome in the CNS. In previous studies, NLRP3 inflammasome suppression with DMF has been reported in diabetes-associated vascular complications (42) and dextran sulfate sodium-induced colitis (28). In the present study, we demonstrated that DMF exerted its protective effects both on the priming and activation step of NLRP3 inflammasome activation.

Oxidative stress and its leading cause, ROS formation, represent major risk factors for numerous neuropsychiatric disorders. Oxidative stress caused by mitochondrial damage and further mtROS formation also contribute to NLRP3 inflammasome activation in the CNS (43). It has been shown that mtROS activates the NLRP3 inflammasome in microglia $(44,45)$ through deubiquitination of NLRP3 and mitochondria released-DNA, a ligand of NLRP3 inflammasome (46). As an anti-oxidative agent, DMF ameliorates mitochondrial damage and ROS formation (47). These effects could be linked to the modulation of anti-oxidative Nrf2 and NF- $\kappa$ B signaling pathways.

DMF is a well-known and widely used Nrf2 activator approved for RRMS. Nrf2 is a transcription factor leading to the activation of protective pathways in the presence of ROS. Upon activation, Nrf2 unbounded from KEAP1, its inhibitor, and upregulated the expression of protective and anti-oxidant ARE genes, including HO-1, Nqo1, Srxn1, Gstp1, and Gclc (48). It has been demonstrated that DMF mediated these effects by oxidizing KEAP1 on cysteine 151 and translocating Nrf2 into the nucleus (49). Additionally, DMF was found to be the best Nrf2 
A

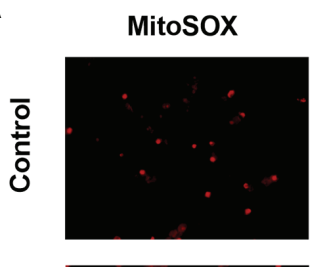

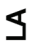
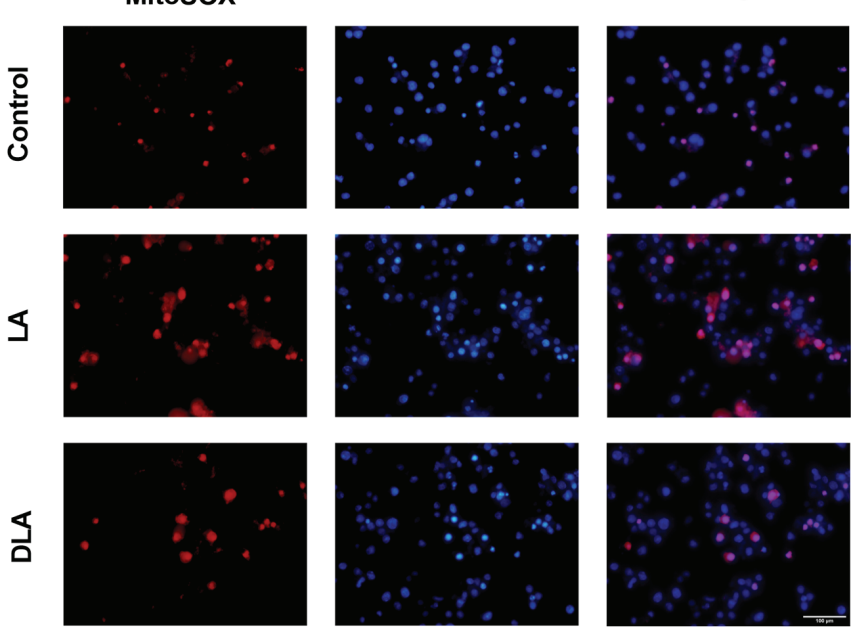

D

Control

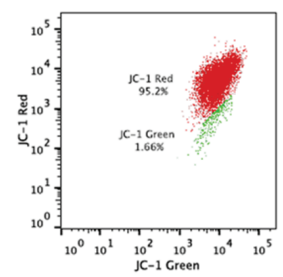

E

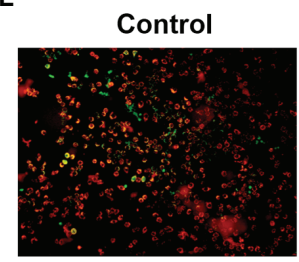

LA

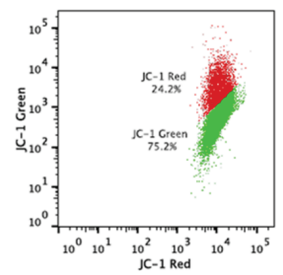

LA

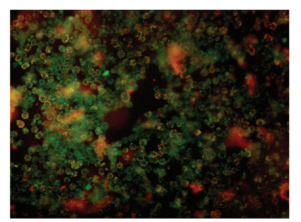

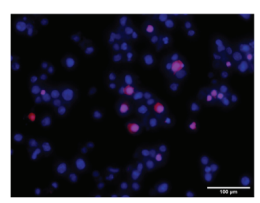

DLA

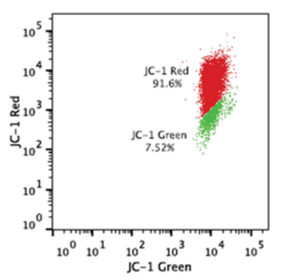

DLA

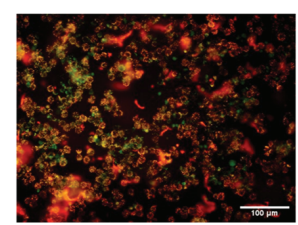

B

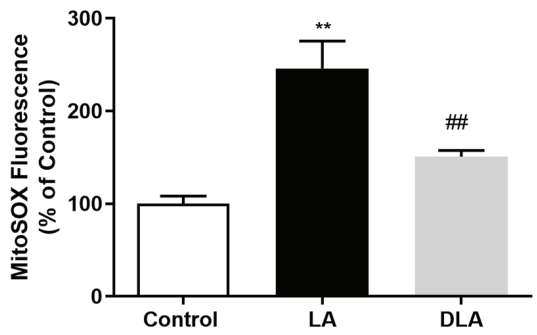

C

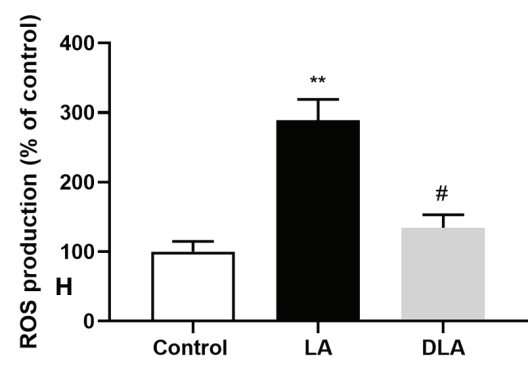

F

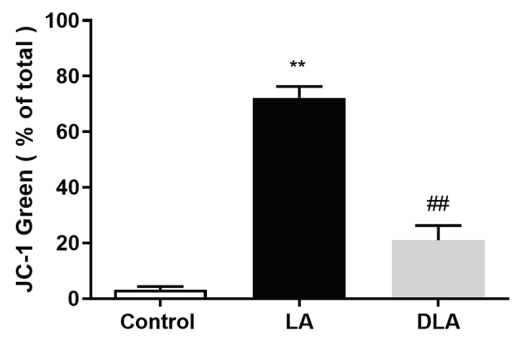

FIGURE 4 | DMF decreased intracellular and mitochondrial ROS formation and restored mitochondrial membrane potential ( $\Delta \Psi \mathrm{m})$. N9 microglia were pretreated with DMF $(10 \mu \mathrm{M})$ for 1 hour, primed with UP-LPS (1000 ng/ml) for 4 hours, and followed by ATP $(5 \mathrm{mM})$ for 1 hour. The anti-oxidative feature of DMF led to mitigated mitochondrial and intracellular ROS formation and balanced mitochondrial membrane polarization $(\Delta \Psi \mathrm{m})$. (A, B) The effects of DMF on mitochondrial ROS formation were evaluated with MitoSOX IF staining and MitoSOX fluorometric assay (Red staining- MitoSOX, Blue staining -Hoechst). (C) The intracellular ROS level was measured with a cellular ROS assay. (D-F) Changes in mitochondrial membrane potential were detected with JC-1 staining via Flow cytometry and IF staining (Red and Green Staining - JC-1). Data are presented as mean \pm S.E.M, $n=5 .{ }^{* \star} p<0.01$ compared to control and ${ }^{\#} \mathrm{p}<0.05$, ${ }^{\# \#} \mathrm{p}<0.01$ compared to LPS and ATP induced cells.

activator among all other fumaric acid esters (50). The beneficial effects of DMF are also attributed to its ability to modulate the NF- $\kappa \mathrm{B}$ pathway. It directly prevents the priming step of NLRP3 inflammasome activation by inhibiting the NF- $\mathrm{KB}$ pathway. Moreover, DMF indirectly inhibits NLRP3 inflammasome activation via the $\mathrm{NF}-\kappa \mathrm{B}$ pathway as the latter pathway is known to be a redox-sensitive regulator resulting to the expression of pro-oxidant enzymes, including NADPH oxidases NOX2 and COX2 (51). Lastly, the crosstalk between Nrf2 and NF- $\kappa B$ pathways might be increasing the potency of DMF's anti-oxidative properties as upregulation of $\mathrm{HO} 1$ prevents the NF- $\kappa \mathrm{B}$ pathway (52). Our study showed that
DMF reversed mitochondrial damage and inhibited cellular ROS and mtROS formation, indicating DMF's mitoprotective properties. DMF also inhibited the NF- $\kappa \mathrm{B}$ pathway and downregulated expression of inflammatory miRNAs, miR-155 and miR-146a, suggesting that DMF induces differential expression of miRNAs, and therefore its protective nature might be substantially related to miRNAs. Furthermore, DMF induced the Nrf2 pathway and the use of ML385, specific Nrf2 inhibitor, confirmed DMF's protective effects on NLRP3 inflammasome activation mediated by Nrf2. In the ML385administered group, the reduced levels of NLRP3 and caspase1 were reversed with DMF treatments. Therefore, our in vivo 

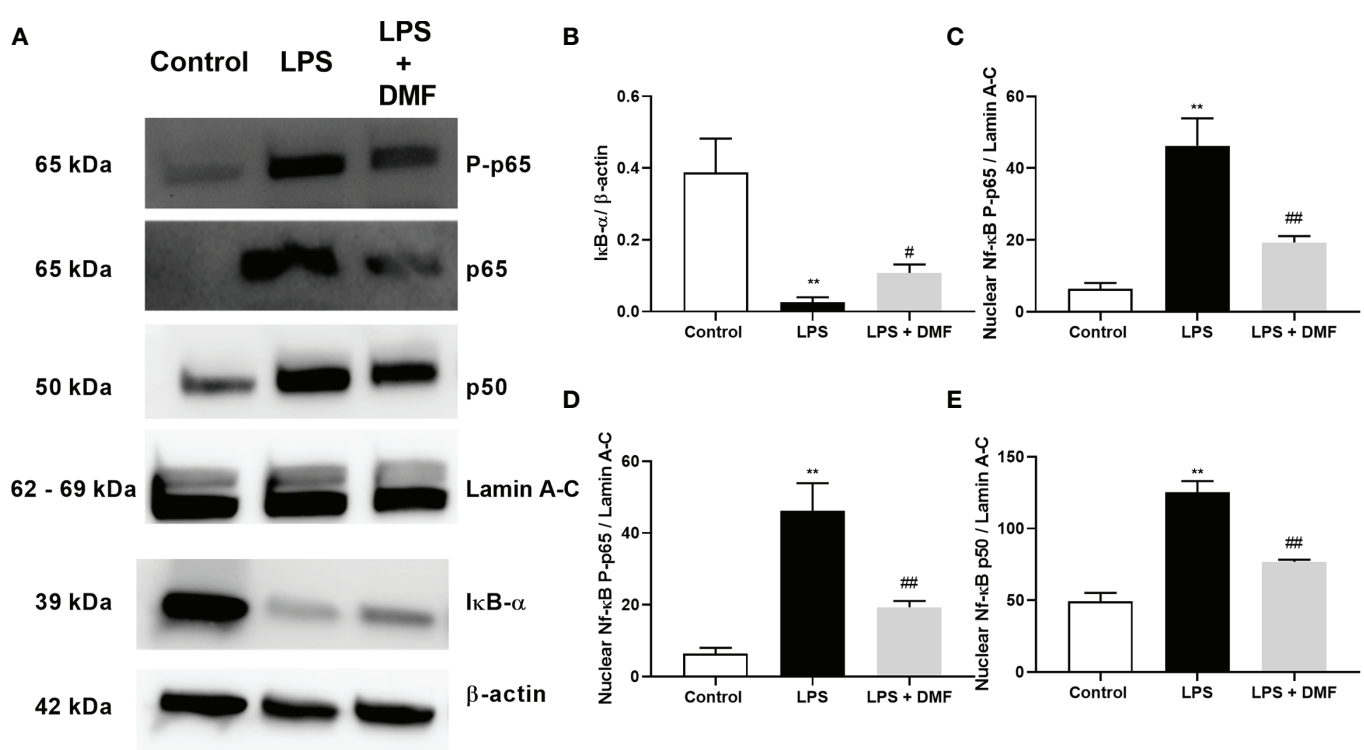

F

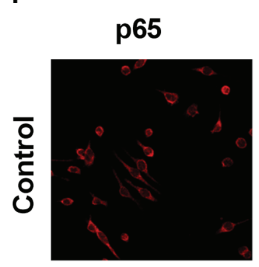

$$
\text { Hoechst }
$$
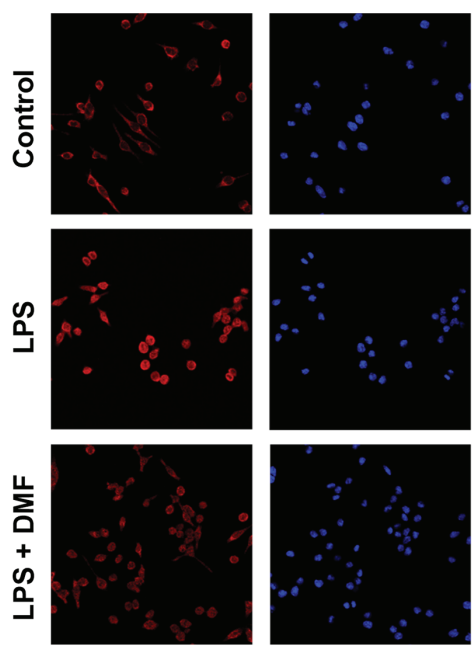

H

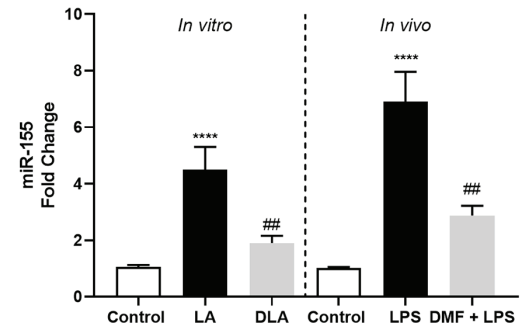

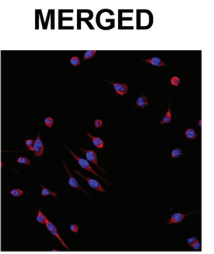
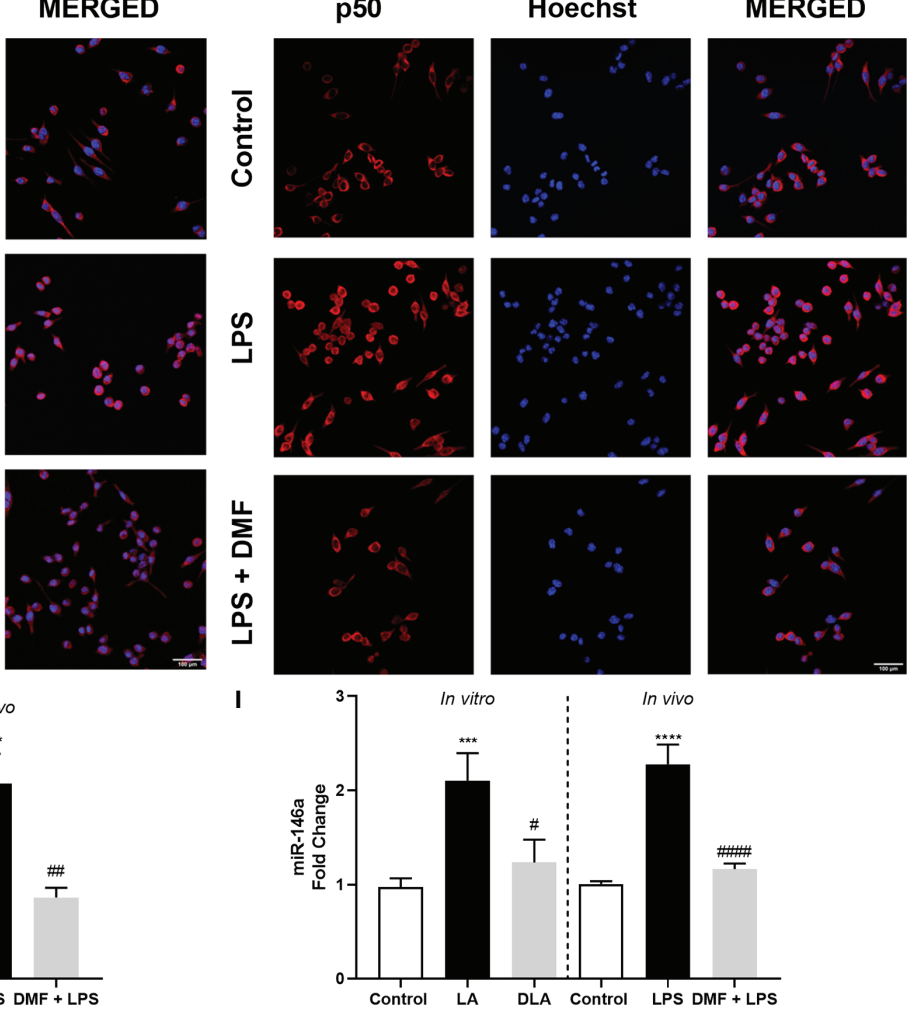

FIGURE 5 | DMF prevented the translocation of NF- $\mathrm{KB}$ into the nucleus and altered miR-155 and miR-146a expression. N9 microglia were pretreated with DMF (10 $\mu \mathrm{M})$ for 1 hour, primed with UP-LPS (1000 ng/ml) for 1 hour. DMF modulated NF- $\mathrm{BB}$ activity by inhibiting the translocation of p-p65, p65, and p50 subunits of NF- $\mathrm{kB}$ protein complex into the nucleus (A). The expression level of $1 \kappa B-\alpha(\mathbf{B})$, translocation of p-p65 (C), p65 (D), and p50 (E) subunits of NF- $\kappa B$ were demonstrated with Western blot. Translocation of p65 (F) and p50 (G) subunits of NF- $\mathrm{KB}$ was demonstrated by IF staining (Red staining - NF- $\mathrm{KB}$ subunits, Blue staining - Hoechst). DMF also downregulated the expression of miR-155 and miR-146a. Expression levels of (H) miR-155 and (I) miR-146 in LPS+ATP treated N9 microglia and LPS challenged mice were measured with RT-qPCR. Data are presented as mean \pm S.E.M, $n=5 .{ }^{* \star} p<0.01,{ }^{\star \star \star} p<0.001,{ }^{\star \star \star \star} p<0.0001$ compared to control and

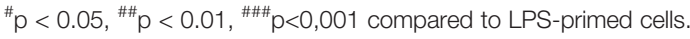


A
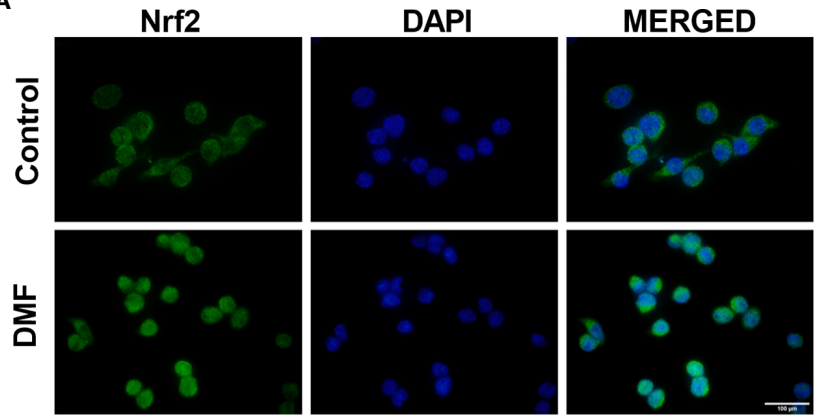

C

Control DMF 1h DMF 3h

$100 \mathrm{kDa}$
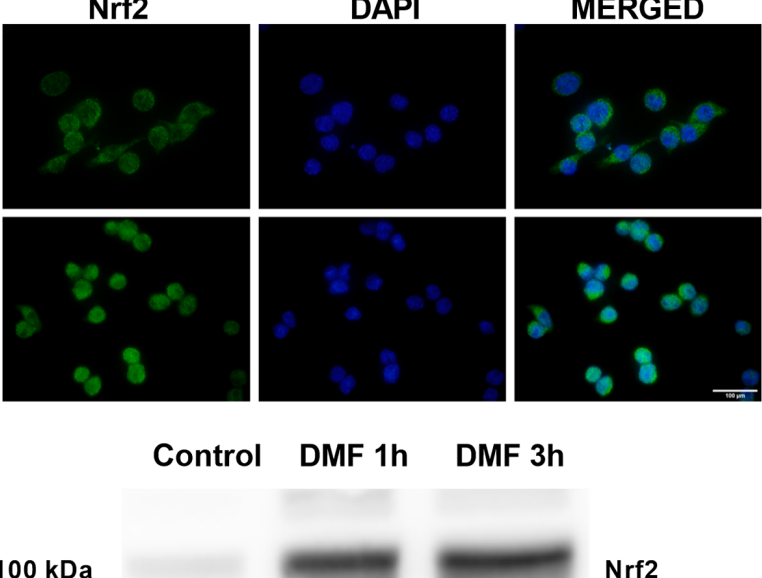

Nrf2

$62-69 \mathrm{kDa}$

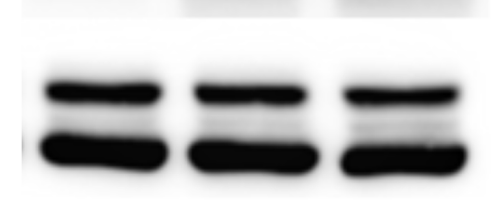

Lamin A-C
B

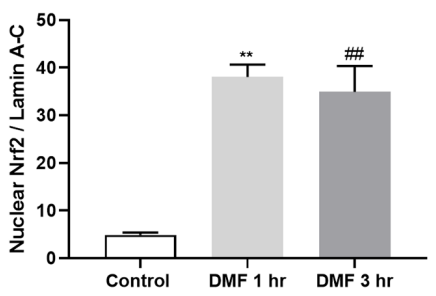

D

\begin{tabular}{lll} 
Genes & Fold Change & p-Value \\
\hline Ho-1 & 31,77 & 0,0043 \\
Nqo1 & 27,62 & 0,0022 \\
Gclc & 3,01 & 0,0079 \\
Gclm & 22,53 & 0,0022 \\
Keap-1 & 1,86 & 0,0159 \\
Srxn1 & 21,44 & 0,0079
\end{tabular}

E
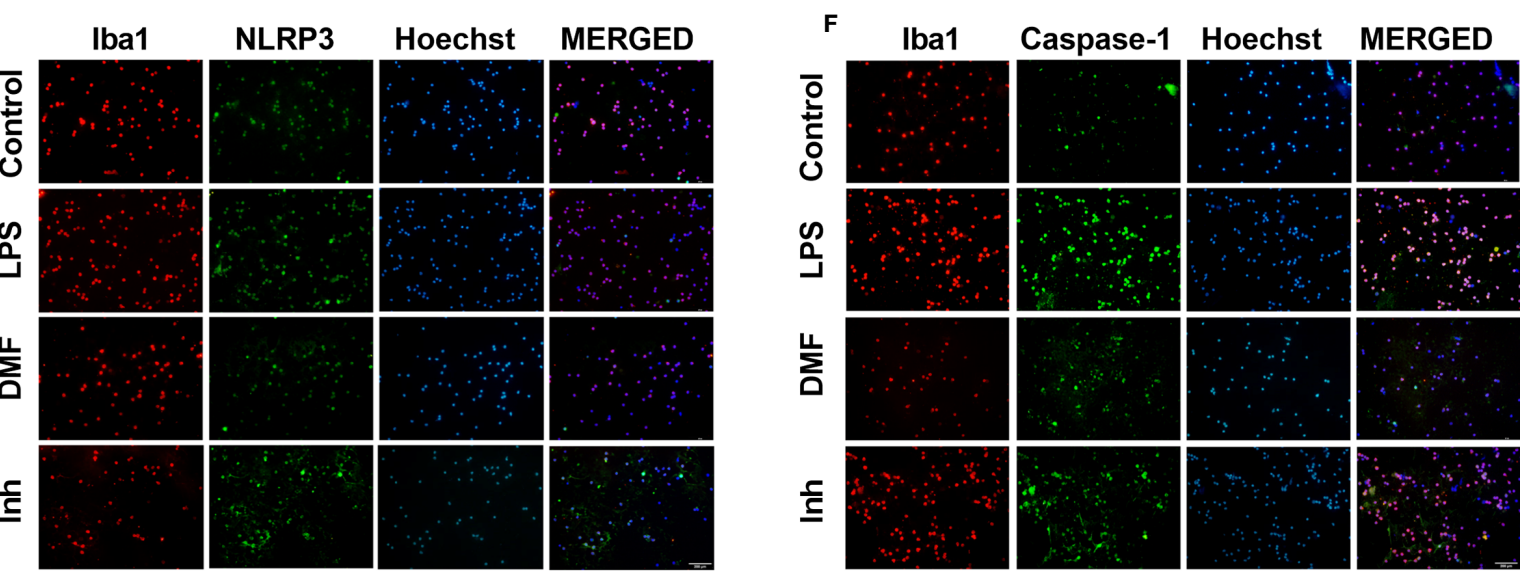

G

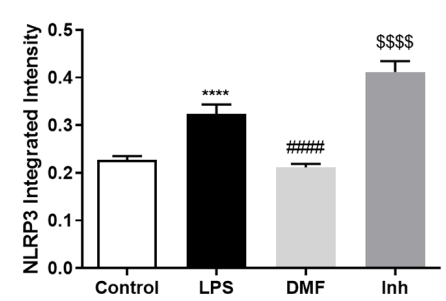

H

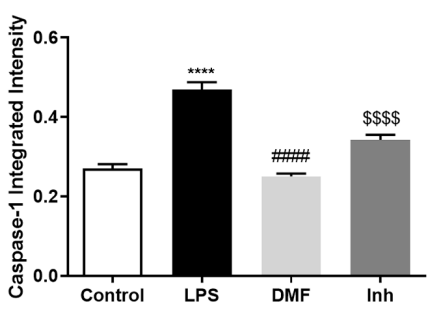

FIGURE 6 | DMF induced Nrf2 activity, and inhibition of Nrf2 activity ameliorated the protective effects of DMF against microglial NLRP3 inflammasome activation. $\mathrm{N} 9$ microglia were treated with DMF $(10 \mu \mathrm{M})$ for $1 \mathrm{~h}$ or $3 \mathrm{~h}$. DMF induced the translocation of transcription factor Nrf2 into the nucleus and induced expression of Nrf2 target genes. (A, C, D) Translocation of Nrf2 was demonstrated with IF staining (Green staining - Nrf2, Blue staining - Hoechst) and Western blot. (B) mRNA levels of Nrf2 target genes were measured with qRT-PCR. (E-H) DMF reduced NLRP3 and Caspase-1 expression in microglia isolated from LPS-challenged mice (Red staining - Iba1, Green staining - Nlrp3/Caspase-1, Blue staining - Hoechst). Data are presented as mean \pm S.E.M, $n=5 .{ }^{\star \star} p<0.01,{ }^{\star \star \star \star} \mathrm{p}<0.0001$ compared to control and ${ }^{\# \#} \mathrm{p}<0.01$, \#\#\#\# $<00,0001$ compared to LPS-challenged cells; ${ }^{\$ \$ \$} \mathrm{p}<0,0001$ compared to DMF pretreated cells. 
A

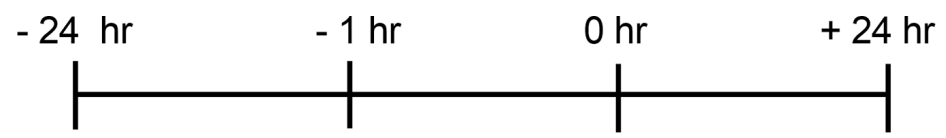
ML- 385
DMF
LPS $(5 \mathrm{mg} / \mathrm{Kg}$ )
Behavioral Tests
$(30 \mathrm{mg} / \mathrm{Kg}$ )
(30 mg/Kg)
Administration
\& Sacrification \&
Pretreatment
Pretreatment
Tissue Collection

B

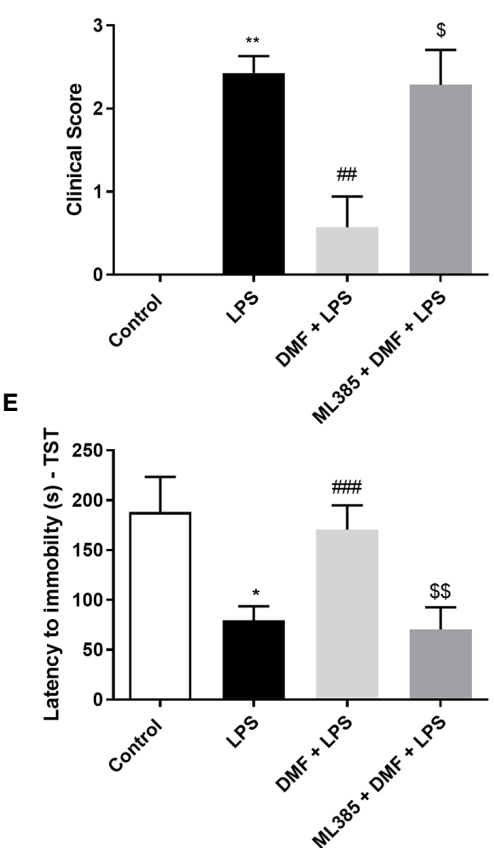

c

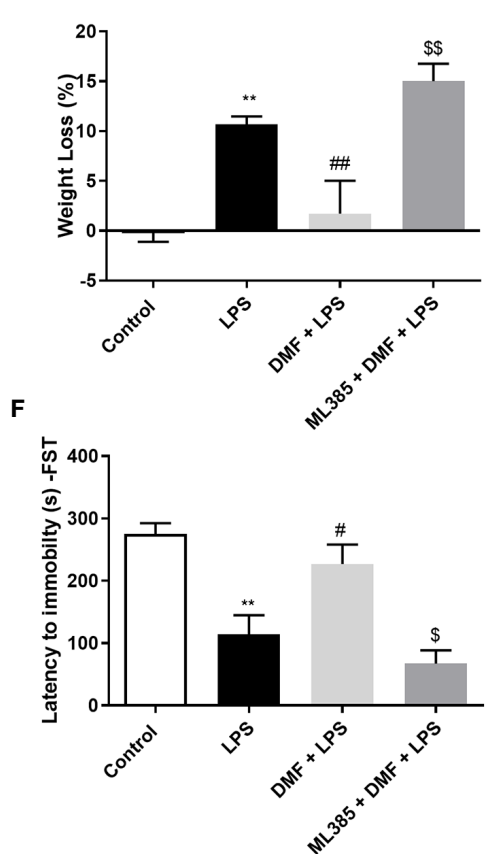

D

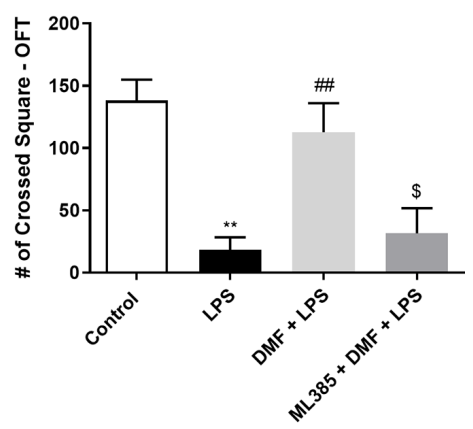

FIGURE 7 | DMF alleviated sickness behaviors in LPS-challenged mice. DMF administration to LPS-challenged mice prevented weight loss and improved clinical and behavioral scores. (A) A schematic representation of the study design (B) Clinical evaluation of mice ( $n=7 /$ group) and (C) Changes in the weight of mice. Assessment of sickness behaviors was conducted with OFT (D), TST (E), and FST (F). Data are presented as mean \pm S.E.M, $n=5$. ${ }^{\star} p<0,05$, ${ }^{\star *} p<0.01$ compared to control, ${ }^{\#} \mathrm{p}<0.05,{ }^{\# \#} \mathrm{p}<0.01$, ${ }^{\# \# \#} \mathrm{p}<0,001$ compared to LPS-challenged mice, ${ }^{\$} \mathrm{p}<0,05,{ }^{\$ \$} \mathrm{p}<0,01$ compared to DMF-administered mice.

results indicated that DMF treatment had protective effects on LPS challenged mice via Nrf2 signaling.

Although we have demonstrated DMF's protective effects on microglial NLRP3 inflammasome activation, our study has certain limitations. First, our in vivo study was conducted only in male mice. Sex-specific differences have been reported in microglia (53-55), and these differences may play a critical role in the alteration in the immune response. Thus, confirmation of our results in a mixed-sex study group in mice is required. Second, the focus of the current study is the microglial NLRP3 inflammasome. Although microglia are primary innate immune cells in the CNS, astrocytes express NLRP3 and other inflammasomes and participate in the immune response along with microglia $(56,57)$. Lastly, systemic inflammation caused by LPS administration in mice was not evaluated in the current study. Although we measured the clinical score and changes in body weight in LPS-challenged mice, other parameters and their implications for microglial activation were not examined.

\section{CONCLUSION}

Altogether, our findings demonstrated for the first time that DMF exerted protective properties on microglial NLRP3 inflammasome activation and subsequent pyroptotic cell death (Figure 8). As it is widely known that NLRP3 inflammasome activation contributes to numerous neurodegenerative and neuropsychiatric disorders, our study brings new insights into how DMF modulates NLRP3 inflammasome activation and lays the foundation for the 


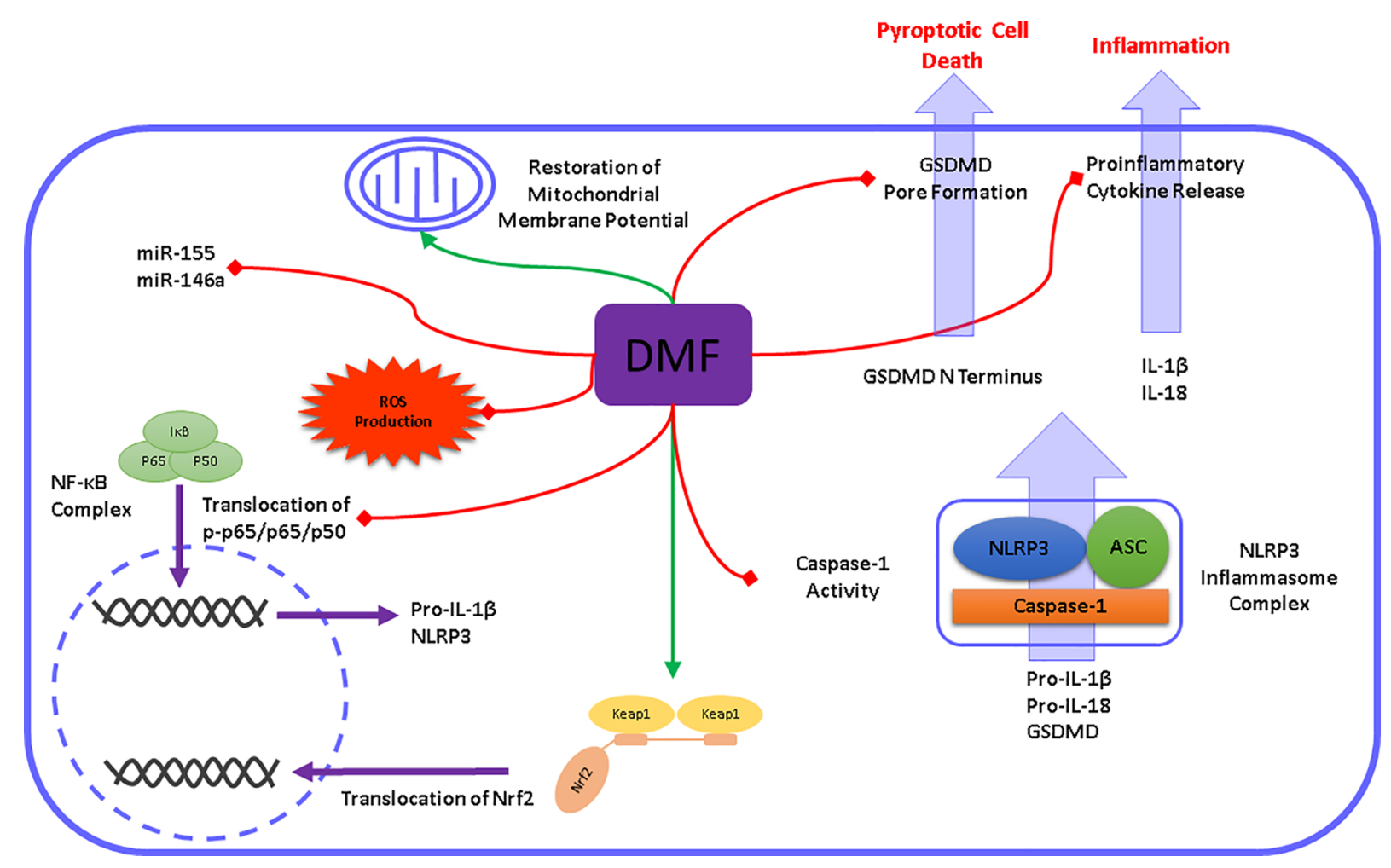

FIGURE 8 | Schematic illustration of DMF modulating microglial NLRP3 inflammasome activation. DMF modulates the NLRP3 inflammasome signaling pathway at different levels. In the priming step, DMF prevents the translocation of NF- $\kappa B$ and subsequently downregulates the expression of NLRP3, IL-1 $\beta$, and IL-18. Additionally, DMF promotes Nrf2 activation and upregulates the expression of Nrf2 target genes. Accordingly, DMF reduced the formation of cellular and mitochondrial ROS and restored the mitochondrial membrane potential. In the activation step, DMF inhibits caspase-1 cleavage and its activity. Subsequent cleavage of pro-inflammatory cytokines and pore-forming GSDMD was prevented by DMF.

development of novel strategies against conditions like LPSinduced sickness behavior.

\section{DATA AVAILABILITY STATEMENT}

The original contributions presented in the study are included in the article/Supplementary Material. Further inquiries can be directed to the corresponding author.

\section{ETHICS STATEMENT}

The animal study was reviewed and approved by Izmir International Biomedicine and Genome Institute Local Ethic Committee for Animal Experiments.

\section{AUTHOR CONTRIBUTIONS}

BT, KG, and SG designed the study. BT, BIA, KUT, ET, CPG designed and performed the experiments. BT, BIA, KUT, ET, $\mathrm{KG}$, and SG analyzed and interpreted the data. BT, BIA, KG, and
SG wrote the manuscript. All authors contributed to the article and approved the submitted version.

\section{FUNDING}

The study was funded by The Scientific and Technological Research Council of Turkey (TUBITAK, Project No: 215Z473) and Dokuz Eylul University Department of Scientific Research Projects (DEU-BAP, Project No: 2017.KB.SAG.039).

\section{ACKNOWLEDGMENTS}

The authors would like to thank Asst. Prof. Dr. Athanasia Pavlopoulou for critical reading of the manuscript; Prof. Dr. Pembe Keskinoglu for her contribution to statistics; and Dr. Melis Olcum, IBG vivarium, and IBG Optical Imaging Core facility members for their contributions.

\section{SUPPLEMENTARY MATERIAL}

The Supplementary Material for this article can be found online at: https://www.frontiersin.org/articles/10.3389/fimmu.2021. 737065/full\#supplementary-material 


\section{REFERENCES}

1. Shabab T, Khanabdali R, Moghadamtousi SZ, Kadir HA, Mohan G. Neuroinflammation Pathways: A General Review. Int J Neurosci (2017) 127 (7):624-33. doi: 10.1080/00207454.2016.1212854

2. Streit WJ, Mrak RE, Griffin WS. Microglia and Neuroinflammation: A Pathological Perspective. J Neuroinflamm (2004) 1(1):14. doi: 10.1186/ 1742-2094-1-14

3. Basu Mallik S, Mudgal J, Nampoothiri M, Hall S, Dukie SA, Grant G, et al. Caffeic Acid Attenuates Lipopolysaccharide-Induced Sickness Behaviour and Neuroinflammation in Mice. Neurosci Lett (2016) 632:218-23. doi: 10.1016/ j.neulet.2016.08.044

4. Aubert A, Goodall G, Dantzer R, Gheusi G. Differential Effects of Lipopolysaccharide on Pup Retrieving and Nest Building in Lactating Mice. Brain Behav Immun (1997) 11(2):107-18. doi: 10.1006/brbi.1997.0485

5. Shattuck EC, Muehlenbein MP. Human Sickness Behavior: Ultimate and Proximate Explanations. Am J Phys Anthropol (2015) 157(1):1-18. doi: 10.1002/ajpa.22698

6. Zhao Z, Wang Y, Zhou R, Li Y, Gao Y, Tu D, et al. A Novel Role of NLRP3Generated IL-1beta in the Acute-Chronic Transition of Peripheral Lipopolysaccharide-Elicited Neuroinflammation: Implications for SepsisAssociated Neurodegeneration. J Neuroinflamm (2020) 17(1):64. doi: 10.1186/s12974-020-1728-5

7. Schroder K, Tschopp J. The Inflammasomes. Cell (2010) 140(6):821-32. doi: 10.1016/j.cell.2010.01.040

8. Lupfer C, Kanneganti TD. The Expanding Role of NLRs in Antiviral Immunity. Immunol Rev (2013) 255(1):13-24. doi: 10.1111/imr.12089

9. Zhou K, Shi L, Wang Y, Chen S, Zhang J. Recent Advances of the NLRP3 Inflammasome in Central Nervous System Disorders. J Immunol Res (2016) 2016:9238290. doi: 10.1155/2016/9238290

10. Olcum M, Tastan B, Ercan I, Eltutan IB, Genc S. Inhibitory Effects of Phytochemicals on NLRP3 Inflammasome Activation: A Review. Phytomedicine (2020) 75:153238. doi: 10.1016/j.phymed.2020.153238

11. Mangan MSJ, Olhava EJ, Roush WR, Seidel HM, Glick GD, Latz E. Targeting the NLRP3 Inflammasome in Inflammatory Diseases. Nat Rev Drug Discov (2018) 17(8):588-606. doi: 10.1038/nrd.2018.97

12. Bauernfeind FG, Horvath G, Stutz A, Alnemri ES, MacDonald K, Speert D, et al. Cutting Edge: NF-kappaB Activating Pattern Recognition and Cytokine Receptors License NLRP3 Inflammasome Activation by Regulating NLRP3 Expression. J Immunol (2009) 183(2):787-91. doi: 10.4049/jimmunol.0901363

13. Herman FJ, Pasinetti GM. Principles of Inflammasome Priming and Inhibition: Implications for Psychiatric Disorders. Brain Behav Immun (2018) 73:66-84. doi: 10.1016/j.bbi.2018.06.010

14. Lamkanfi M, Kanneganti TD. Nlrp3: An Immune Sensor of Cellular Stress and Infection. Int J Biochem Cell Biol (2010) 42(6):792-5. doi: 10.1016/ j.biocel.2010.01.008

15. Prochnicki T, Latz E. Inflammasomes on the Crossroads of Innate Immune Recognition and Metabolic Control. Cell Metab (2017) 26(1):71-93. doi: 10.1016/j.cmet.2017.06.018

16. Yang Y, Wang H, Kouadir M, Song H, Shi F. Recent Advances in the Mechanisms of NLRP3 Inflammasome Activation and Its Inhibitors. Cell Death Dis (2019) 10(2):128. doi: 10.1038/s41419-019-1413-8

17. Lu F, Lan Z, Xin Z, He C, Guo Z, Xia X, et al. Emerging Insights Into Molecular Mechanisms Underlying Pyroptosis and Functions of Inflammasomes in Diseases. J Cell Physiol (2020) 235(4):3207-21. doi: 10.1002/jcp.29268

18. Blair HA. Dimethyl Fumarate: A Review in Relapsing-Remitting Ms. Drugs (2019) 79(18):1965-76. doi: 10.1007/s40265-019-01229-3

19. Fox RJ, Miller DH, Phillips JT, Hutchinson M, Havrdova E, Kita M, et al. PlaceboControlled Phase 3 Study of Oral BG-12 or Glatiramer in Multiple Sclerosis. N Engl J Med (2012) 367(12):1087-97. doi: 10.1056/NEJMoa1206328

20. Gold R, Kappos L, Arnold DL, Bar-Or A, Giovannoni G, Selmaj K, et al. PlaceboControlled Phase 3 Study of Oral BG-12 for Relapsing Multiple Sclerosis. N Engl J Med (2012) 367(12):1098-107. doi: 10.1056/NEJMoa1114287

21. Diebold M, Sievers C, Bantug G, Sanderson N, Kappos L, Kuhle J, et al. Dimethyl Fumarate Influences Innate and Adaptive Immunity in Multiple Sclerosis. J Autoimmun (2018) 86:39-50. doi: 10.1016/j.jaut.2017.09.009

22. Paraiso HC, Kuo PC, Curfman ET, Moon HJ, Sweazey RD, Yen JH, et al. Dimethyl Fumarate Attenuates Reactive Microglia and Long-Term Memory
Deficits Following Systemic Immune Challenge. J Neuroinflamm (2018) 15 (1):100. doi: 10.1186/s12974-018-1125-5

23. Wang Q, Chuikov S, Taitano S, Wu Q, Rastogi A, Tuck SJ, et al. Dimethyl Fumarate Protects Neural Stem/Progenitor Cells and Neurons From Oxidative Damage Through Nrf2-ERK1/2 MAPK Pathway. Int J Mol Sci (2015) 16(6):13885-907. doi: 10.3390/ijms160613885

24. Lastra D, Fernandez-Gines R, Manda G, Cuadrado A. Perspectives on the Clinical Development of NRF2-Targeting Drugs. Handb Exp Pharmacol (2020) 264:93-141. doi: 10.1007/164_2020_381

25. Cuadrado A, Manda G, Hassan A, Alcaraz MJ, Barbas C, Daiber A, et al. Transcription Factor NRF2 as a Therapeutic Target for Chronic Diseases: A Systems Medicine Approach. Pharmacol Rev (2018) 70(2):348-83. doi: 10.1124/pr.117.014753

26. Afonina IS, Zhong Z, Karin M, Beyaert R. Limiting Inflammation-the Negative Regulation of NF-kappaB and the NLRP3 Inflammasome. Nat Immunol (2017) 18(8):861-9. doi: 10.1038/ni.3772

27. Miglio G, Veglia E, Fantozzi R. Fumaric Acid Esters Prevent the NLRP3 Inflammasome-Mediated and ATP-Triggered Pyroptosis of Differentiated THP-1 Cells. Int Immunopharmacol (2015) 28(1):215-9. doi: 10.1016/ j.intimp.2015.06.011

28. Liu X, Zhou W, Zhang X, Lu P, Du Q, Tao L, et al. Dimethyl Fumarate Ameliorates Dextran Sulfate Sodium-Induced Murine Experimental Colitis by Activating Nrf2 and Suppressing NLRP3 Inflammasome Activation. Biochem Pharmacol (2016) 112:37-49. doi: 10.1016/j.bcp.2016.05.002

29. Righi M, Mori L, De Libero G, Sironi M, Biondi A, Mantovani A, et al. Monokine Production by Microglial Cell Clones. Eur J Immunol (1989) 19 (8):1443-8. doi: 10.1002/eji.1830190815

30. Arioz BI, Tastan B, Tarakcioglu E, Tufekci KU, Olcum M, Ersoy N, et al. Melatonin Attenuates LPS-Induced Acute Depressive-Like Behaviors and Microglial NLRP3 Inflammasome Activation Through the SIRT1/Nrf2 Pathway. Front Immunol (2019) 10:1511. doi: 10.3389/fimmu.2019.01511

31. Schindelin J, Arganda-Carreras I, Frise E, Kaynig V, Longair M, Pietzsch T, et al. Fiji: An Open-Source Platform for Biological-Image Analysis. Nat Methods (2012) 9(7):676-82. doi: 10.1038/nmeth.2019

32. Gandhi R, Hayley S, Gibb J, Merali Z, Anisman H. Influence of Poly I:C on Sickness Behaviors, Plasma Cytokines, Corticosterone and Central Monoamine Activity: Moderation by Social Stressors. Brain Behav Immun (2007) 21(4):477-89. doi: 10.1016/j.bbi.2006.12.005

33. Zoller T, Attaai A, Potru PS, Russ T, Spittau B. Aged Mouse Cortical Microglia Display an Activation Profile Suggesting Immunotolerogenic Functions. Int $J$ Mol Sci (2018) 19(3):706. doi: 10.3390/ijms19030706

34. Place DE, Kanneganti TD. Recent Advances in Inflammasome Biology. Curr Opin Immunol (2017) 50:32-8. doi: 10.1016/j.coi.2017.10.011

35. Mazuco TRR, Biondi TF, Silva EP, Bernardi MM, Kirsten TB. LPS-Induced Sickness Behavior is Not Affected by Selenium But Is Switched Off by Psychogenic Stress in Rats. Vet Res Commun (2019) 43(4):239-47. doi: 10.1007/s11259-019-09766-8

36. Dantzer R. Cytokine, Sickness Behavior, and Depression. Immunol Allergy Clin North Am (2009) 29(2):247-64. doi: 10.1016/j.iac.2009.02.002

37. Stansley B, Post J, Hensley K. A Comparative Review of Cell Culture Systems for the Study of Microglial Biology in Alzheimer's Disease. J Neuroinflamm (2012) 9:115. doi: 10.1186/1742-2094-9-115

38. Peng H, Li H, Sheehy A, Cullen P, Allaire N, Scannevin RH. Dimethyl Fumarate Alters Microglia Phenotype and Protects Neurons Against Proinflammatory Toxic Microenvironments. J Neuroimmunol (2016) 299:35-44. doi: 10.1016/j.jneuroim.2016.08.006

39. Campolo M, Casili G, Biundo F, Crupi R, Cordaro M, Cuzzocrea S, et al. The Neuroprotective Effect of Dimethyl Fumarate in an MPTP-Mouse Model of Parkinson's Disease: Involvement of Reactive Oxygen Species/Nuclear FactorKappab/Nuclear Transcription Factor Related to NF-E2. Antioxid Redox Signal (2017) 27(8):453-71. doi: 10.1089/ars.2016.6800

40. Wilms H, Sievers J, Rickert U, Rostami-Yazdi M, Mrowietz U, Lucius R. Dimethylfumarate Inhibits Microglial and Astrocytic Inflammation by Suppressing the Synthesis of Nitric Oxide, IL-1beta, TNF-Alpha and IL-6 in an in-Vitro Model of Brain Inflammation. J Neuroinflamm (2010) 7:30. doi: 10.1186/1742-2094-7-30

41. Kortam MA, Ali BM, Fathy N. The Deleterious Effect of Stress-Induced Depression on Rat Liver: Protective Role of Resveratrol and Dimethyl 
Fumarate via Inhibiting the MAPK/ERK/JNK Pathway. J Biochem Mol Toxicol (2021) 35(1):e22627. doi: 10.1002/jbt.22627

42. Amin FM, Abdelaziz RR, Hamed MF, Nader MA, Shehatou GSG. Dimethyl Fumarate Ameliorates Diabetes-Associated Vascular Complications Through ROS-TXNIP-NLRP3 Inflammasome Pathway. Life Sci (2020) 256:117887. doi: $10.1016 /$ j.lfs.2020.117887

43. Zhou R, Yazdi AS, Menu P, Tschopp J. A Role for Mitochondria in NLRP3 Inflammasome Activation. Nature (2011) 469(7329):221-5. doi: 10.1038/ nature09663

44. Sarkar S, Malovic E, Harishchandra DS, Ghaisas S, Panicker N, Charli A, et al. Mitochondrial Impairment in Microglia Amplifies NLRP3 Inflammasome Proinflammatory Signaling in Cell Culture and Animal Models of Parkinson's Disease. NPJ Parkinsons Dis (2017) 3:30. doi: 10.1038/s41531-017-0032-2

45. Wang HM, Zhang T, Huang JK, Xiang JY, Chen JJ, Fu JL, et al. Edaravone Attenuates the Proinflammatory Response in Amyloid-Beta-Treated Microglia by Inhibiting NLRP3 Inflammasome-Mediated IL-1beta Secretion. Cell Physiol Biochem (2017) 43(3):1113-25. doi: 10.1159/000481753

46. Liu Q, Zhang D, Hu D, Zhou X, Zhou Y. The Role of Mitochondria in NLRP3 Inflammasome Activation. Mol Immunol (2018) 103:115-24. doi: 10.1016/ j.molimm.2018.09.010

47. Scuderi SA, Ardizzone A, Paterniti I, Esposito E, Campolo M. Antioxidant and Anti-Inflammatory Effect of Nrf2 Inducer Dimethyl Fumarate in Neurodegenerative Diseases. Antioxidants (Basel) (2020) 9(7):630. doi: 10.3390/antiox 9070630

48. Eren E, Tufekci KU, Isci KB, Tastan B, Genc K, Genc S. Sulforaphane Inhibits Lipopolysaccharide-Induced Inflammation, Cytotoxicity, Oxidative Stress, and miR-155 Expression and Switches to Mox Phenotype Through Activating Extracellular Signal-Regulated Kinase 1/2-Nuclear Factor Erythroid 2-Related Factor 2/Antioxidant Response Element Pathway in Murine Microglial Cells. Front Immunol (2018) 9:36. doi: 10.3389/ fimmu.2018.00036

49. Yamamoto M, Kensler TW, Motohashi H. The KEAP1-NRF2 System: A Thiol-Based Sensor-Effector Apparatus for Maintaining Redox Homeostasis. Physiol Rev (2018) 98(3):1169-203. doi: 10.1152/physrev.00023.2017

50. Kourakis S, Timpani CA, de Haan JB, Gueven N, Fischer D, Rybalka E. Dimethyl Fumarate and Its Esters: A Drug With Broad Clinical Utility? Pharmaceuticals (Basel) (2020) 13(10):306. doi: 10.3390/ph13100306

51. Morgan MJ, Liu ZG. Crosstalk of Reactive Oxygen Species and NF-kappaB Signaling. Cell Res (2011) 21(1):103-15. doi: 10.1038/cr.2010.178
52. Wardyn JD, Ponsford AH, Sanderson CM. Dissecting Molecular Cross-Talk Between Nrf2 and NF-kappaB Response Pathways. Biochem Soc Trans (2015) 43(4):621-6. doi: 10.1042/BST20150014

53. Hui CW, Vecchiarelli HA, Gervais E, Luo X, Michaud F, Scheefhals L, et al. Sex Differences of Microglia and Synapses in the Hippocampal Dentate Gyrus of Adult Mouse Offspring Exposed to Maternal Immune Activation. Front Cell Neurosci (2020) 14:558181. doi: 10.3389/fncel.2020.558181

54. Liu LL, Li JM, Su WJ, Wang B, Jiang CL. Sex Differences in Depressive-Like Behaviour may Relate to Imbalance of Microglia Activation in the Hippocampus. Brain Behav Immun (2019) 81:188-97. doi: 10.1016/ j.bbi.2019.06.012

55. Guneykaya D, Ivanov A, Hernandez DP, Haage V, Wojtas B, Meyer N, et al. Transcriptional and Translational Differences of Microglia From Male and Female Brains. Cell Rep (2018) 24(10):2773-83.e6. doi: 10.1016/ j.celrep.2018.08.001

56. Jha MK, Jo M, Kim JH, Suk K. Microglia-Astrocyte Crosstalk: An Intimate Molecular Conversation. Neuroscientist (2019) 25(3):227-40. doi: 10.1177/ 1073858418783959

57. Freeman L, Guo H, David CN, Brickey WJ, Jha S, Ting JP. NLR Members NLRC4 and NLRP3 Mediate Sterile Inflammasome Activation in Microglia and Astrocytes. J Exp Med (2017) 214(5):1351-70. doi: 10.1084/jem.20150237

Conflict of Interest: The authors declare that the research was conducted in the absence of any commercial or financial relationships that could be construed as a potential conflict of interest.

Publisher's Note: All claims expressed in this article are solely those of the authors and do not necessarily represent those of their affiliated organizations, or those of the publisher, the editors and the reviewers. Any product that may be evaluated in this article, or claim that may be made by its manufacturer, is not guaranteed or endorsed by the publisher.

Copyright $\odot 2021$ Tastan, Arioz, Tufekci, Tarakcioglu, Gonul, Genc and Genc. This is an open-access article distributed under the terms of the Creative Commons Attribution License (CC BY). The use, distribution or reproduction in other forums is permitted, provided the original author(s) and the copyright owner(s) are credited and that the original publication in this journal is cited, in accordance with accepted academic practice. No use, distribution or reproduction is permitted which does not comply with these terms. 